BULLETIN (New Series) OF THE

AMERICAN MATHEMATICAL SOCIETY

Volume 50, Number 1, January 2013, Pages 57-92

S 0273-0979(2012)01393-9

Article electronically published on October 11, 2012

\title{
THE COBORDISM HYPOTHESIS
}

\author{
DANIEL S. FREED
}

\begin{abstract}
In this expository paper we introduce extended topological quantum field theories and the cobordism hypothesis.
\end{abstract}

\section{INTRODUCTION}

The cobordism hypothesis was conjectured by Baez and Dolan $\mathrm{BD}$ in the mid1990s. It has now been proved by Hopkins and Lurie in dimension two and by Lurie in higher dimensions. There are many complicated foundational issues which lie behind the definitions and the proof, and only a detailed sketch [L1] has appeared so far 11 The history of the Baez-Dolan conjecture goes most directly through quantum field theory and its adaptation to low-dimensional topology. Yet in retrospect it is a theorem about the structure of manifolds in all dimensions, and at the core of the proof lies Morse theory. Hence there are two routes to the cobordism hypothesis: algebraic topology and quantum field theory.

Consider the abelian group $\Omega_{0}^{S O}$ generated by compact oriented 0-dimensional manifolds, that is, finite sets $Y$ of points each labeled with + or - . The group operation is disjoint union. We deem $Y_{0}$ equivalent to $Y_{1}$ if there is a compact oriented 1-manifold $X$ with oriented boundary $Y_{1} \amalg-Y_{0}$. Then a basic theorem in differential topology [Mi1, Appendix] asserts that $\Omega_{0}^{S O}$ is the free abelian group with a single generator, the positively oriented point $\mathrm{pt}_{+} 2$ This result is the cornerstone of smooth intersection theory. From the point of view of algebraic topology, the cobordism hypothesis is a similar statement about a more ornate structure built from smooth manifolds. The simplest version is for framed manifolds. The language is off-putting if unfamiliar, and it will be explained in due course.

Theorem 1.1 (Cobordism hypothesis: heuristic algebro-topological version). For $n \geq 1$, $\operatorname{Bord}_{n}^{\mathrm{fr}}$ is the free symmetric monoidal $(\infty, n)$-category with duals generated by $\mathrm{pt}_{+}$.

Received by the editors November 15, 2011 and in revised form September 14, 2012.

2010 Mathematics Subject Classification. Primary 57R56.

The work of this author was supported by the National Science Foundation under grant DMS0603964 .

${ }^{1}$ Nonetheless, we use "theorem" and its synonyms in this manuscript. The foundations are rapidly being filled in and alternative proofs have also been carried out, though none has yet appeared in print.

${ }^{2}$ Two important remarks: (1) we can replace orientations with framings; (2) for unoriented manifolds the group $\Omega_{0}^{O}$ is not free on one generator, but rather there is a relation and $\Omega_{0}^{O} \cong \mathbb{Z} / 2 \mathbb{Z}$. 
The "Bord" in Bord ${ }_{n}^{\text {fr }}$ stands for "bordism" 3 and pt + is now the point with the standard framing. Bord ${ }_{n}^{\text {fr }}$ is an elaborate algebraic gadget which encodes $n$-framed manifolds with corners of dimensions $\leq n$, and it tracks gluings and disjoint unions. One of our goals is to motivate this elaborate algebraic structure.

An extended topological field theory is a representation of the bordism category, i.e., a homomorphism $F$ : $\operatorname{Bord}_{n}^{\text {fr }} \rightarrow \mathcal{C}$. The codomain $\mathcal{C}$ is a symmetric monoidal $(\infty, n)$-category, typically linear in nature. In important examples $F$ assigns a complex number to every closed $n$-manifold and a complex vector space to every closed $(n-1)$-manifold.

Theorem 1.2 (Cobordism hypothesis: weak quantum field theory version). $A$ homomorphism $F: \operatorname{Bord}_{n}^{\mathrm{fr}} \rightarrow \mathcal{C}$ is determined by $F\left(\mathrm{pt}_{+}\right)$.

The object $F\left(\mathrm{pt}_{+}\right) \in \mathcal{C}$ satisfies stringent finiteness conditions expressed in terms of dualities, and the real power of the cobordism hypothesis is an existence statement: if $x \in \mathcal{C}$ is $n$-dualizable, then there exists a topological field theory $F$ with $F\left(\mathrm{pt}_{+}\right)=x$. Precise statements of the cobordism hypothesis appear in $\sqrt[6]{6}$,

Our plan is to build up gradually to the categorical complexities inherent in extended field theories and the cobordism hypothesis. So in the next two sections we take strolls along the two routes to the cobordism hypothesis: algebraic topology (\$2) and quantum field theory (\$3). Section 4 is an extended introduction to nonextended topological field theory. The simple examples discussed there only hint at the power of this circle of ideas. In $\$ 5$ we turn to extended field theories and so also to higher categories. The cobordism hypothesis is the subject of $₫ 6$, where we state a complete version in Theorem 6.8. The cobordism hypothesis connects in exciting ways to other parts of topology, geometry, and representation theory as well as to some contemporary ideas in quantum field theory. A few of these are highlighted in 97

The manuscript [L1] has leisurely introductions to higher categorical ideas and to the setting of the cobordism hypothesis, in addition to a detailed sketch of the proof and applications. The original paper $[\mathrm{BD}$ is another excellent source of expository material. Additional recent expositions are available in [L3] and [Te1]. We have endeavored to complement these expositions rather than duplicate them. I warmly thank David Ben-Zvi, Andrew Blumberg, Lee Cohn, Tim Perutz, Ulrike Tillmann, and the referee for their comments and suggestions.

\section{Algebraic topology}

The most basic maneuvers in algebraic topology extract algebra from spaces. For example, to a topological space $X$ we associate a sequence of abelian groups $\left\{H_{q}(X)\right\}$. There are several constructions of these homology groups, but for nice spaces they are all equivalent $[\mathrm{Sp}$. The homology construction begins to have teeth only when we tell how homology varies with $X$. One elementary assertion is that if $X \simeq Y$ are homeomorphic spaces, then the homology groups are isomorphic. Thus numerical invariants of homology groups, such as the rank, are homeomorphism invariants of topological spaces: Betti numbers. But it is much more powerful to remember the isomorphisms of homology groups associated to homeomorphisms, and indeed the homomorphisms associated to arbitrary continuous maps. This is

\footnotetext{
3 "Bordism" replaces the older "cobordism", as bordism is part of homology whereas cobordism is part of cohomology A1.
} 
naturally encoded in the algebraic structure of a category. Here is an informal definition; see standard texts (e.g., $\underline{\mathrm{Mc}}$ ) for details.

Definition 2.1. A category $\mathcal{C}$ consists of a set $C_{0}$ of objects $\{x\}$, a set $C_{1}$ of morphisms $\{f: x \rightarrow y\}$, identity elements $\left\{i d_{x}: x \rightarrow x\right\}$, and an associative composition law $f, g \longmapsto g \circ f$ for morphisms $x \stackrel{f}{\rightarrow} y$ and $y \stackrel{g}{\rightarrow} z$. If $\mathcal{C}, \mathcal{D}$ are categories, then a homomorphism $5: \mathcal{C} \rightarrow \mathcal{D}$ is a pair $\left(F_{0}, F_{1}\right)$ of maps of sets $F_{i}: \mathcal{C}_{i} \rightarrow \mathcal{D}_{i}$ which preserves compositions.

More formally, there are source and target maps $C_{1} \rightarrow C_{0}$, identity elements are defined by a map $C_{0} \rightarrow C_{1}$, and composition is a map from a subset of $C_{1} \times C_{1}$ to $C_{1}$ - the subset consists of pairs of morphisms for which the target of the first equals the source of the second. A homomorphism also preserves the source and target maps. Topological spaces comprise the objects of a category Top whose morphisms are continuous maps; abelian groups comprise the objects of a category $\mathrm{Ab}$ whose morphisms are group homomorphisms. Some basic properties of homology groups are summarized by the statement that

$$
H_{q}:(\mathrm{Top}, \amalg) \longrightarrow(\mathrm{Ab}, \oplus)
$$

is a homomorphism. We explain $\amalg$ and $\oplus$ in the next paragraph.

The homomorphism property does not nearly characterize homology, and we can encode many more properties via extra structure on Top and Ab. We single out one here - an additional operation on objects and morphisms. If $X_{1}, X_{2}$ are topological spaces, there is a new space $X_{1} \amalg X_{2}$, the disjoint union. The operation $X_{1}, X_{2} \mapsto$ $X_{1} \amalg X_{2}$ has properties analogous to a commutative, associative composition law on a set. For example, the empty set $\emptyset$ is an identity for disjoint union in the sense that $\emptyset \amalg X$ is canonically identified with $X$ for all topological spaces $X$. Furthermore, if $f_{i}: X_{i} \rightarrow Y_{i}, i=1,2$, are continuous maps, there is an induced continuous map $f_{1} \amalg f_{2}: X_{1} \amalg X_{2} \rightarrow Y_{1} \amalg Y_{2}$ on the disjoint union. An operation on a category with these properties is called a symmetric monoidal structure, in this case on the category Top. Similarly, the category Ab of abelian groups has a symmetric monoidal structure given by direct sum: $A_{1}, A_{2} \rightarrow A_{1} \oplus A_{2}$. The homology maps (2.2) are homomorphisms of symmetric monoidal categories: there is a canonical identification of $H_{q}\left(X_{1} \amalg X_{2}\right)$ with $H_{q}\left(X_{1}\right) \oplus H_{q}\left(X_{2}\right)$.

Remark 2.3. Homology is classical in that disjoint unions map to direct sums. We will see that a characteristic property of quantum systems is that disjoint unions map to tensor products. The passage from classical to quantum is (poetically) a passage from addition to multiplication, a kind of exponentiation.

Our interest here is not in all topological spaces, but rather in smooth manifolds. Fix a positive integer $n$.

Definition 2.4. Let $Y_{0}, Y_{1}$ be smooth compact $(n-1)$-dimensional manifolds without boundary. A bordism from $Y_{0}$ to $Y_{1}$ is a compact $n$-dimensional manifold $X$ with boundary, a decomposition $\partial X=\partial X_{0} \amalg \partial X_{1}$, and diffeomorphisms $Y_{i} \rightarrow \partial X_{i}, i=1,2$.

\footnotetext{
${ }^{4}$ We do not worry about technicalities of set theory in this expository paper.

"The word "functor" is usually employed here, but "homomorphism" is more consistent with standard usage elsewhere in algebra.
} 


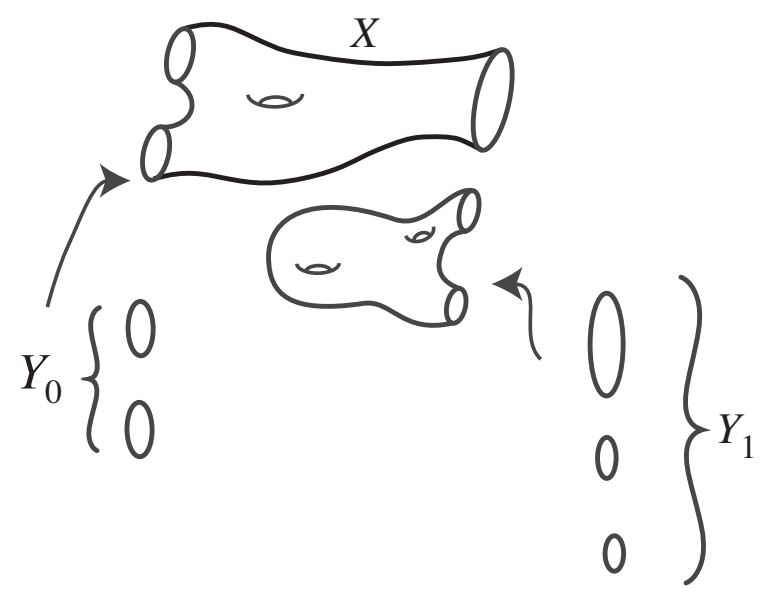

Figure 1. A bordism $X: Y_{0} \rightarrow Y_{1}$

Figure 1 depicts an example which emphasizes that manifolds need not be connected. The empty set $\emptyset$ is a manifold of any dimension. So a closed $n$-manifoldthat is, a compact manifold without boundary - is a bordism from $\emptyset^{n-1}$ to $\emptyset^{n-1}$. Note also that the disjoint union of smooth manifolds is a smooth manifold, and the disjoint union of bordisms is a bordism.

To turn bordism into algebra, we observe that bordism defines an equivalence relation: closed $(n-1)$-manifolds $Y_{0}, Y_{1}$ are bordant if there exists a bordism from $Y_{0}$ to $Y_{1}$. (Observe that to prove transitivity it is convenient to modify Definition 2.4 so that boundary identifications are between the manifolds $[0,1) \times Y_{0},(-1,0] \times Y_{1}$ and open collar neighborhoods of $\partial X_{0}, \partial X_{1}$ : smooth functions glue nicely on open sets.) Disjoint union defines an abelian group structure on the set $\Omega_{n-1}^{O}$ of equivalence classes. For example, $\Omega_{0}^{O} \cong \mathbb{Z} / 2 \mathbb{Z}$ is generated by a single point. Twice a point is the disjoint union of two points, and as two points bound a closed interval, two points are bordant to the empty 0 -manifold. Life is more interesting when we consider manifolds with extra topological structure. For example, there are bordism groups $\Omega_{q}^{S O}$ of oriented manifolds. An orientation on a 0 -manifold consisting of a single point is a choice of + or - . Then $\Omega_{0}^{S O} \cong \mathbb{Z}$ by the map which sends a finite set of oriented points to the number of positive points minus the number of negative points. This is a foundational result in differential topology which enables oriented counts in intersection theory [Mi1. Another interesting structure is a stable framing. It arises in the Pontrjagin-Thom construction (see Figure 2). Let $f: S^{q+N} \rightarrow S^{N}$ be a smooth map. By Sard's theorem there is a regular value $p \in$ $S^{N}$, whence $M:=f^{-1}(p) \subset S^{q+N}$ is a smooth $q$-dimensional submanifold. Also, a basis of $T_{p} S^{N}$ pulls back under $f$ to a global framing of the normal bundle to $M$ in $S^{N}$. If we deform $p$ to another regular value, then the framed manifold $M$ undergoes a bordism. The same is true if $f$ deforms to a smoothly homotopic map. The precise correspondence works in the stable limit $N \rightarrow \infty$ : the stably framed bordism group $\Omega_{q}^{\mathrm{fr}}$ is isomorphic to the stable homotopy group of the sphere $\lim _{N \rightarrow \infty} \pi_{q+N}\left(S^{N}\right)$. This is the most basic link between bordism and homotopy theory. 


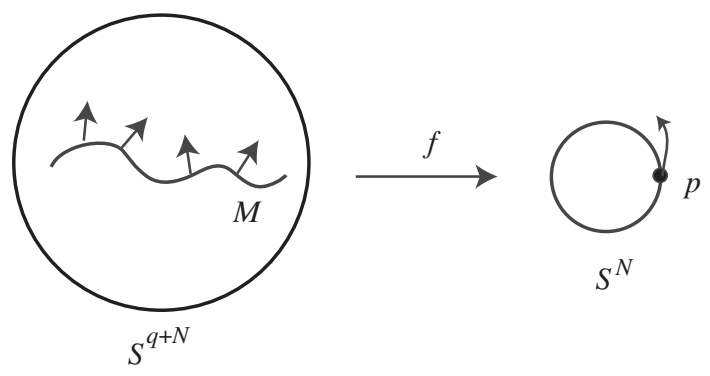

Figure 2. The Pontrjagin-Thom construction

Bordism has a long history in algebraic topology. By 1950 it appears that Pontrjagin had defined abelian groups based on the notion of a bordism, though it was Thom [T] who made the first systematic computations of bordism groups using homotopy theory. There are many variations according to the type of manifold: oriented, spin, framed, etc. Theory and computation of bordism groups were an important part of algebraic topology in the 1950s and 1960s, and they found applications in other parts of topology and geometry. For example, Hirzebruch's 1954 proof of the Riemann-Roch theorem was based on bordism computations, as was the first proof of the Atiyah-Singer index theorem $\mathrm{Pa}$ in 1963.

The bordism group of $d$-dimensional manifolds arises when $(d+1)$-dimensional bordisms are used to define an equivalence relation. Disjoint union of $d$-manifolds gives the abelian group structure. One lesson from classical algebraic topology is that the passage from Betti numbers to homology groups is very fruitful. The analog here is to track bordisms between closed manifold, not merely to observe their existence - in our "categorified" world we encode the bordism as a map. Segal Se2] introduced a bordism category of Riemann surfaces in his axiomatization of 2-dimensional conformal field theory, which inspired Atiyah A2 to axiomatize topological field theories in any dimensions using bordism categories of smooth manifolds with no continuous geometric structure (such as a metric or conformal structure). Tillmann [Til1, Til2] observed that the classifying space of the bordism category, which has the abelian group-like operation of disjoint union, is a spectrum in the sense of stable homotopy theory. Together with Madsen [MT] they conjecturally identify the classifying spectrum of an enriched bordism category - a step towards the $\infty$-categories we meet in $\$ 5$-and show that their conjecture implies Mumford's conjecture $\mathrm{Mu}$ about the rational cohomology of the mapping class group. The Madsen-Tillmann conjecture was subsequently proved in MW] and is now known as the Madsen-Weiss theorem. The relation with the spectra Thom used to compute bordism groups is elucidated in GMTW, §3], where another proof is given.

For now we restrict our discussion to manifolds with boundary-no cornersand so organize closed $(n-1)$-manifolds into a symmetric monoidal category which refines the abelian group $\Omega_{n-1}$.

\footnotetext{
${ }^{6}$ According to $\left.\mathrm{May}, \S 6\right]$ a 1950 Russian paper of Pontrjagin contains bordism groups; see [P] for a later account. Thom [T] also cites work of Rohlin relevant to computations of bordism in low dimensions, but we do not know if Rohlin phrased them in terms of bordism groups.
} 


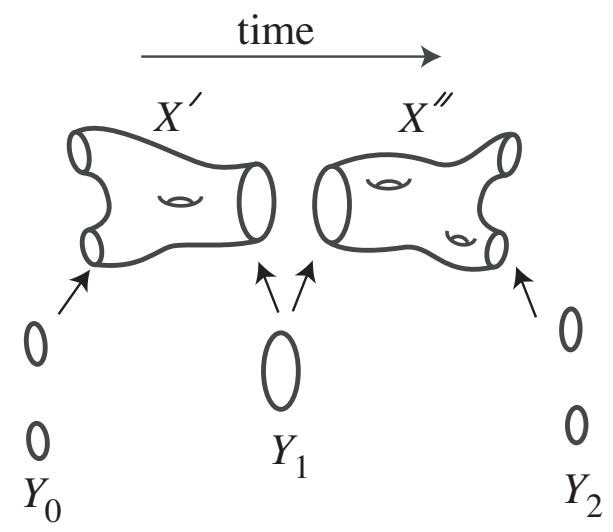

Figure 3. Composition of bordisms

Definition 2.5. $\operatorname{Bord}_{\langle n-1, n\rangle}$ is the symmetric monoidal category whose objects are compact $(n-1)$-manifolds and in which a morphism $X: Y_{0} \rightarrow Y_{1}$ is a bordism from $Y_{0}$ to $Y_{1}$, up to diffeomorphism. The monoidal structure is disjoint union.

So now a bordism is a map - a morphism in a category - and the boundary is divided into incoming (domain) boundary components and outgoing (codomain) boundary components. Heuristically, we say there is an "arrow of time", at least at the boundary; in pictures we draw a global arrow of time. Composition (Figure (3) is defined by gluing bordisms. We identify diffeomorphic bordisms - the diffeomorphism must commute with the boundary identifications - and so obtain a strictly associative composition law. The identity morphism $Y \rightarrow Y$ is the cylinder $[0,1] \times Y$ with obvious boundary identifications. There are variants $\operatorname{Bord}_{\langle n-1, n\rangle}^{S O}$ and Bord $_{\langle n-1, n\rangle}^{\text {fr }}$ for oriented and framed manifolds, but with one important change: in Bord $_{\langle n-1, n\rangle}^{\text {fr }}$ the morphisms $X$ carry framings of the tangent bundle (not stabilized) and the objects $Y$ carry framings of $(1) \oplus T Y$, where "(1)" here denotes the trivial real line bundle of rank one. Notice the contrast: traditional Pontrjagin-Thom theory has stable framings of the normal bundle, whereas $\operatorname{Bord}_{\langle n-1, n\rangle}^{\mathrm{fr}}$ has unstable framings of the tangent bundle.

By analogy to the homology homomorphism (2.2) we are led to the following definition.

Definition 2.6 ([A2]). An $n$-dimensional topological field theory is a homomorphism

$$
F: \operatorname{Bord}_{\langle n-1, n\rangle} \longrightarrow(\mathrm{Ab}, \otimes)
$$

of symmetric monoidal categories.

As telegraphed in Remark 2.3, in a quantum field theory disjoint unions map to tensor products, not direct sums. There are many variations on this definition. The domain can be a bordism category of smooth manifolds with extra structure, or even of singular manifolds. The codomain may be replaced by any symmetric monoidal category, algebraic or not. We introduce a more drastic variant of Definition 2.6 in 95 . A typical choice for the codomain is $\left(\right.$ Vect $\left._{\mathbb{C}}, \otimes\right)$, the category of complex 


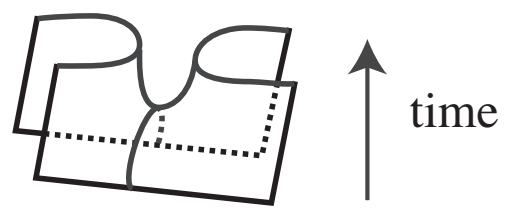

FiguRE 4. An elementary bordism

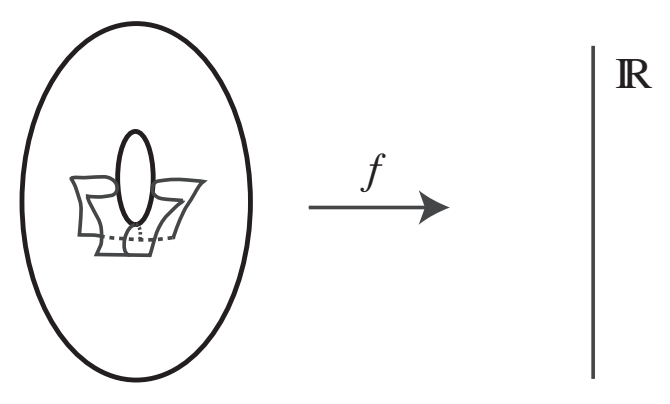

Figure 5. A Morse function

vector spaces under tensor product. A topological field theory with values in Vect $_{\mathbb{C}}$ is a linearization - a linear representation - of manifolds.

We have been led naturally to Definition 2.6 by combining basic ideas in homology and bordism. But this is hardly the historical path! For that we turn in the next section to notions in quantum field theory. Before leaving bordism, though, we pause to remind the reader of the connection with Morse theory.

Intuitively, a Morse function refines the arrow of time to a particular time function. Let $X: Y_{0} \rightarrow Y_{1}$ be an $n$-dimensional bordism. A function $f: X \rightarrow \mathbb{R}$ is compatible with the bordism structure if there exist $t_{0}<t_{1}$ such that $t_{0}, t_{1}$ are regular values of $f$, the image of $f$ is contained in $\left[t_{0}, t_{1}\right]$, and $Y_{i}=f^{-1}\left(t_{i}\right)$. Furthermore, $f$ is a Morse function if it has finitely many isolated nondegenerate critical points. The main theorems in Morse theory [Mi2] assert that slices $f^{-1}(t)$ and $f^{-1}\left(t^{\prime}\right)$ are diffeomorphic if there are no critical values between $t$ and $t^{\prime}$, and at an isolated critical point there is a topology change which is described by a standard surgery. For example, in Figure 4 the local slice evolves from the two parallel line segments at the bottom to the two curves at the top; the saddle depicts the elementary bordism which connects the two local slices. Figure 5 displays the standard example of a Morse function on the torus - the height function - and embeds the elementary bordism of Figure 4 into a neighborhood of one of the critical points of index 1.

Remark 2.8. The local description of the topology change at a critical point uses a manifold with corners, as in Figure 4. Manifolds with boundary and no corners do not suffice. The additional locality afforded by admitting corners - and eventually higher codimensional corners - is a crucial idea for the cobordism hypothesis; see $\$ 5$,

Morse functions exist as a consequence of Sard's theorem. This means that any bordism can be decomposed as a composition of elementary bordisms, one for each critical point. Manipulations with Morse functions are a key ingredient in Milnor's presentation [Mi3] of Smale's $h$-cobordism theorem $[\mathrm{Sm}$. The space of Morse functions on a fixed bordism has many components: Morse functions in 
different components induce qualitatively different decompositions into elementary bordisms. Cerf [C] relaxed the Morse condition to construct a connected space of functions. This enables a systematic study of transitions between decompositions. For example, Cerf theory is the basis for Kirby calculus [K], which describes links in 3-manifolds and 4-manifolds. As we shall see, it is also a crucial tool for constructing topological field theories.

An elementary illustrative example of a Cerf transition is the family of functions

$$
f_{t}(x)=\frac{x^{3}}{3}-t x, \quad x, t \in \mathbb{R} .
$$

For $t>0$ this is a Morse function with nondegenerate critical points at $x= \pm \sqrt{t}$. For $t<0$ it is a Morse function with no critical points. At $t=0$ the function fails to be Morse: $x=0$ is a degenerate critical point. So as $t$ increases from negative to positive, two critical points are born on the $x$-line, and they separate at birth. In the other direction, as $t$ decreases from positive to negative, the two critical points collide and annihilate. This simple "birth-death transition" is all that is needed to connect different components of Morse functions.

\section{QuANTUM FIELD THEORY}

For much of its history quantum field theory was tied to four space-time dimensions and a handful of physically realistic examples. As opposed to quantum mechanics, where the underlying theory of Hilbert spaces and operator theory has been fully developed, the analytic underpinnings of quantum field theory remain unsettled. Still, there has been a huge transformation over the past three decades. Quantum field theorists now study a large set of examples in a variety of dimensions, not all of which are meant to be physically relevant. A deeper engagement with mathematicians and mathematics has led physicists to study models whose consequences are more relevant to geometry than to accelerators. Topological and algebraic aspects of quantum field theories have come to the fore. From another direction string theory has illuminated the subject, and there are new ties to condensed matter theory as well.

In this section we briefly sketch how Definition 2.6 of a topological quantum field theory emerges from physics. Our exposition is purely formal, extracting the structural elements which most directly lead to our goal. Let us begin with quantum mechanics, which is a 1-dimensional quantum field theory. (The dimension of a theory refers to space-time, and at least in mainstream theories there is a single time dimension. Thus a 1-dimensional theory only has time; space is treated externally.) The basic ingredients are a complex separable Hilbert space $\mathscr{H}$ and for each time interval of length $t$ a unitary operator

$$
U_{t}=e^{-i t H / \hbar} .
$$

Here $H: \mathscr{H} \rightarrow \mathscr{H}$ is the self-adjoint Hamiltonian which describes the quantum system, and $\hbar$ is Planck's constant. The pure states of the system are vectors (really complex lines of vectors) in $\mathscr{H}$, and the unitary operators (3.1) describe the evolution of a state in time. Self-adjoint operators $\mathcal{O}$ on $\mathscr{H}$ act on the system - they are the observables - and the physics is encoded in expectation values

$$
\left\langle\Omega, U_{t_{n}} \mathcal{O}_{n} \cdots U_{t_{2}} \mathcal{O}_{2} U_{t_{1}} \mathcal{O}_{1} U_{t_{0}} \Omega\right\rangle .
$$




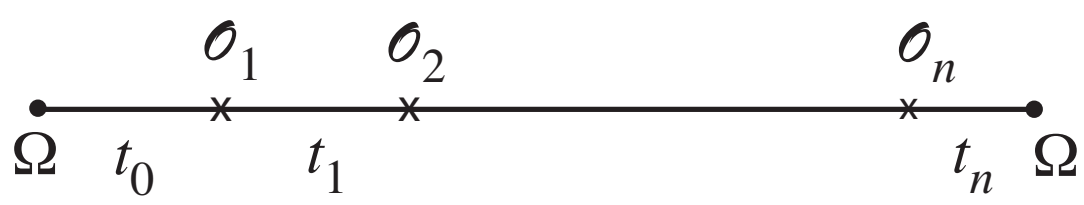

Figure 6. Vacuum expectation value in quantum mechanics

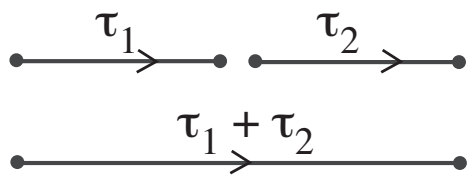

Figure 7. Composition of 1-dimensional bordisms

In this expression the state $\Omega$ evolves for time $t_{0}$, is acted on by the operator $\mathcal{O}_{1}$, then evolves for time $t_{1}$, then is acted on by the operator $\mathcal{O}_{2}$, etc. See Figure 6 for a pictorial representation. We recommend $\mathrm{Ma}, \mathrm{Fa}$, for structural expositions of mechanics which elucidate the pairing of states and observables.

It is convenient and powerful to analytically continue time $t$ from the real line to the complex line with the restriction $\operatorname{Im} t<0$. Real times are now at the boundary of allowed complex times. If the Hamiltonian $H$ is nonnegative and $\operatorname{Im} t<0$, then the evolution operator $e^{-i t H / \hbar}$ is a contracting operator. Wick rotation to imaginary time is the further restriction to purely imaginary $t=\tau / \sqrt{-1}$, where the Euclidean time $\tau$ is strictly positive. We associate the Euclidean contracting evolution $F_{\tau}=e^{-\tau H / \hbar}$ to an interval of length $\tau$, that is, to a compact, connected Riemannian 1-manifold with boundary whose total length is $\tau$. The evolution obeys a semigroup law

$$
F_{\tau_{2}+\tau_{1}}=F_{\tau_{2}} \circ F_{\tau_{1}},
$$

as illustrated in Figure 7 This is already reminiscent of bordism. We can imagine a bordism category Bord ${ }_{\langle 0,1\rangle}^{\text {Riem }}$ whose objects are compact oriented 0-manifolds and whose morphisms are compact Riemannian oriented 1-manifolds with boundary. The semigroup law for the evolution of a quantum mechanical system is encoded in the statement that

$$
F: \operatorname{Bord}_{\langle 0,1\rangle}^{\text {Riem }} \longrightarrow \text { Hilb }
$$

is a homomorphism to the category of Hilbert spaces and contracting linear maps. Notice that $F$ encodes more than evolution. For example, we demand that $F$ be a homomorphism of symmetric monoidal categories mapping disjoint unions to tensor products, which encodes the idea that the state space of the union of quantum mechanical systems is a tensor product. Exotic "evolutions" are now possible; see Figure 8. In a more careful axiomatization [Se1 one takes the codomain to be a category of topological vector spaces; then the Hilbert space structure emerges more organically from the geometry, as do the operator insertions in (3.2).

It is a small step now to pass from the formal description (3.4) of a quantum mechanical system to the assertion that an $n$-dimensional quantum field theory is 


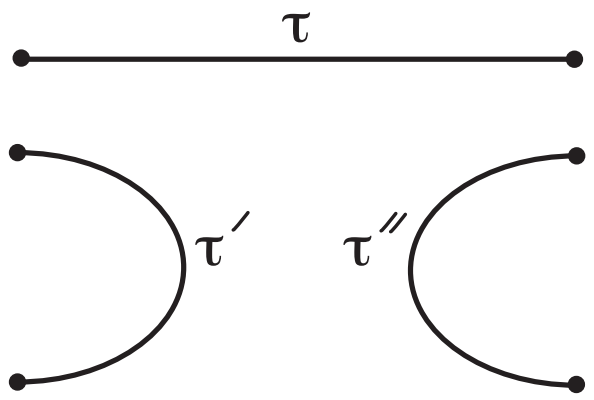

FIGURE 8. Exotic evolutions in quantum mechanics

a homomorphism

$$
F: \operatorname{Bord}_{\langle n-1, n\rangle}^{\text {Riem }} \longrightarrow \text { Hilb }
$$

from the bordism category of Riemannian $n$-dimensional bordisms ("Riemannian space-times") to the category of Hilbert spaces (better: topological vector spaces). If $X$ is such a bordism, and $x \in X$ a point not on the boundary, then the boundary sphere of the geodesic ball of sufficiently small radius $r$ maps under $F$ to a topological vector space $\mathscr{H}_{r}$, and the limit as $r \rightarrow 0$ is a topological vector space of operators associated to the point $x$. We can approximate it by the topological vector space at some small finite radius $r_{0}$. Remove an open ball of radius $r_{0}$ about $x$. Choose the arrow of time so that the new boundary component - the sphere of radius $r_{0}$ about $x$-is incoming. For example, the bordism in Figure 9 has incoming boundary $Y_{0}$ union the spheres about $x_{1}, x_{2}$, and $x_{3}$ and outgoing boundary $Y_{1}$. A field theory $F$ determines topological vector spaces $F\left(Y_{0}\right), F\left(Y_{1}\right)$ for the boundary components and then topological vector spaces $V_{1}, V_{2}, V_{3}$ associated to the points $x_{1}, x_{2}, x_{3}$. The bordism $X$ goes over to a linear map

$$
F(X): V_{1} \otimes V_{2} \otimes V_{3} \longrightarrow \operatorname{Hom}\left(F\left(Y_{0}\right), F\left(Y_{1}\right)\right) .
$$

This is the sense in which the topological vector spaces $V_{i}$ attach a space of operators to $x_{i}$, analogously to the operators which appear in (3.2) as illustrated in

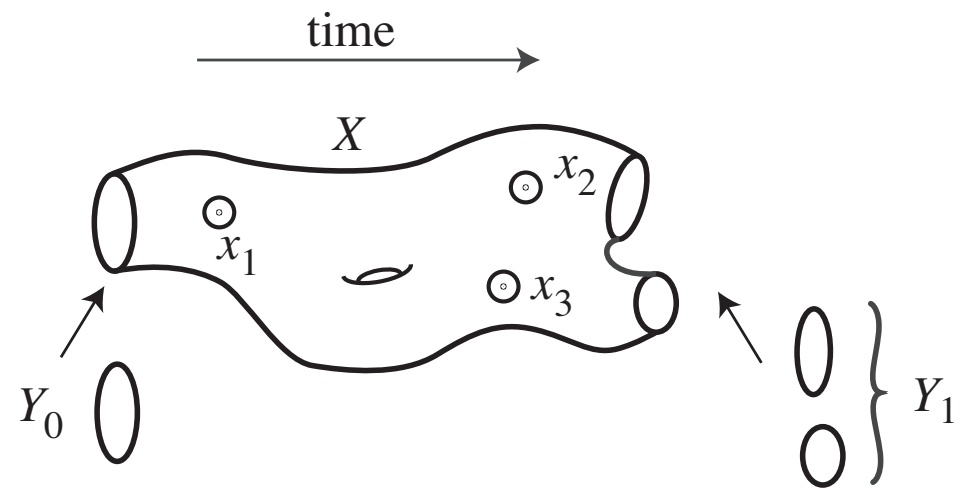

Figure 9. Operator insertions 
Figure 6] In case $Y_{0}=Y_{1}=\emptyset^{n-1}$, then $F(X)$ is called a correlation function between "operators" at the points $x_{i}$. If in addition there are no points $x_{i}$, then $F(X)$ is a complex number, the partition function of the closed manifold $X$.

This geometric formulation of quantum field theory, developed in the 1980s out of the interaction between mathematicians and physicists, centered around 2-dimensional conformal field theory. Graeme Segal's samizdat manuscript, The definition of conformal field theory (now published [Se2]), was widely distributed and very influential among both mathematicians and physicists. Segal's recent series of lectures [Se1] explores and expands on these ideas in the context of general quantum field theories. More traditional mathematical treatments of quantum field theory [SW], [H], GJ] are set in 4-dimensional Minkowski space-time and focus on analytic aspects. The geometric formulation set the stage for the advent of topological field theories. In 1988 Witten W1 introduced twistings of supersymmetric quantum field theories on Minkowski space-time which allow them to be formulated on arbitrary oriented Riemannian manifolds. Special correlation functions in twisted theories are topological invariants. Witten's first application was to a supersymmetric gauge theory in four dimensions - a theory whose principal field is a connection on a principal bundle - where he showed that Donaldson's polynomial invariants of 4-manifolds [D] are correlation functions in that twisted supersymmetric gauge theory. Two-dimensional supersymmetric $\sigma$-models - whose principal field is a map $\Sigma \rightarrow M$ from a 2-manifold into a Riemannian target manifold - also admit topological twistings in case there is enough supersymmetry (which constrains the target manifold to be Kähler in the basic case). These 2-dimensional topological field theories W2 have had profound consequences for algebraic geometry in the form of Gromov-Witten invariants and mirror symmetry. By late 1988 Witten realized [W3] that the Jones polynomials of knots and links in $S^{3}$ are encoded in a 3-dimensional field theory-called Chern-Simons theory after the classical action functional of connections which defines it - and he used it to introduce new invariants of 3-manifolds. This theory, as opposed to the topologically twisted supersymmetric models, is topological at the classical level and has an immediate connection to combinatorially accessible invariants. For many mathematicians it served as an accessible entrée into quantum field theory. In early 1989 Atiyah A2 introduced a set of axioms for topological quantum field theory which essentially amount to Definition 2.6.

\section{TOPOLOGICAL QUANTUM FIELD THEORY}

In this section we flesh out Definition 2.6 for simple 1-dimensional and 2-dimensional theories. The constructions and theorems give a taste of what is possible in more complicated and interesting situations. We include a rigorous finite version of the Feynman path integral; the nonrigorous infinite version is one of the main tools in a quantum field theorist's arsenal.

1-dimensional theories. Let us begin our exploration of Definition 2.6 with a 1dimensional topological field theory of oriented manifolds. Recall that the domain of such a theory is the bordism category $\operatorname{Bord}_{\langle 0,1\rangle}^{S O}$ in which an object is a compact oriented 0-manifold - a finite set of points, each with a + or - attached - and a morphism is an oriented 1-dimensional bordism. In more detail, if $X: Y_{0} \rightarrow Y_{1}$ is an oriented bordism, then $X$ is a manifold with boundary, and so at $x \in \partial X$ we 
have a short exact sequence of real vector spaces

$$
0 \longrightarrow T_{x} \partial X \longrightarrow T_{x} X \longrightarrow T_{x} X / T_{x} \partial X \longrightarrow 0 .
$$

The normal bundle carries a canonical orientation: vectors which exponentiate to curves leaving the manifold are positively oriented. However, when interpreted as a bordism we use the arrow of time to orient the normal bundle. Namely, outgoing boundary components have the canonical orientation and incoming boundary components the opposite to the canonical orientation. Then using (4.1) an orientation of $X$ induces one on $\partial X$, and we require that the diffeomorphisms $Y_{i} \rightarrow \partial X_{i}$ in Definition 2.4 preserve the induced orientation. There is a time-reversal operation which reverses the arrow of time (swaps incoming and outgoing), hence the orientation of the normal bundle at the boundary and so too the induced boundary orientation.

There are two basic objects in $\operatorname{Bord}_{\langle 0,1\rangle}^{S O}$ : the + point and the - point. Any other object is a tensor product (disjoint union) of these. Some basic morphisms are illustrated in Figure 10. The arrow of time points to the right, whereas the orientation is notated by an arrow on each component of the bordism. Notice that there is a correlation between the orientation, the arrow of time, and the boundary orientation. The first two morphisms are identities. The third is called coevaluation and the fourth evaluation. The second bordism is obtained from the first by time-reversal, and the same holds for the third and fourth bordisms. In this case time reversal is a duality operation: the - point is the dual of the + point and the evaluation is dual to the coevaluation. The coevaluation and evaluation are evolutions in 1-dimensional topological field theory which go beyond the standard evolutions in quantum mechanics (Figure 8). Also, in quantum mechanics the closed intervals are Riemannian, so have a length $\tau$, whereas in the topological theory all closed intervals are diffeomorphic and lead to the identity evolution. Comparison with (3.1) shows that the Hamiltonian vanishes in a topological field theory. There is no local evolution: all of the nonidentity behavior comes from topology.

Now suppose $F$ is a 1-dimensional oriented topological field theory (2.7) with values in complex vector spaces:

$$
F:\left(\operatorname{Bord}_{\langle 0,1\rangle}^{S O}, \amalg\right) \longrightarrow\left(\text { Vect }_{\mathbb{C}}, \otimes\right) .
$$

The notation recalls that $F$ is a homomorphism of symmetric monoidal categories, so it maps disjoint unions to tensor products. The homomorphism $F$ assigns a vector space $F\left(\mathrm{pt}_{+}\right)=V_{+}$to the + point and a vector space $F\left(\mathrm{pt}_{-}\right)=V_{-}$to the - point. This determines the value of $F$ on all compact oriented 0 -manifolds as

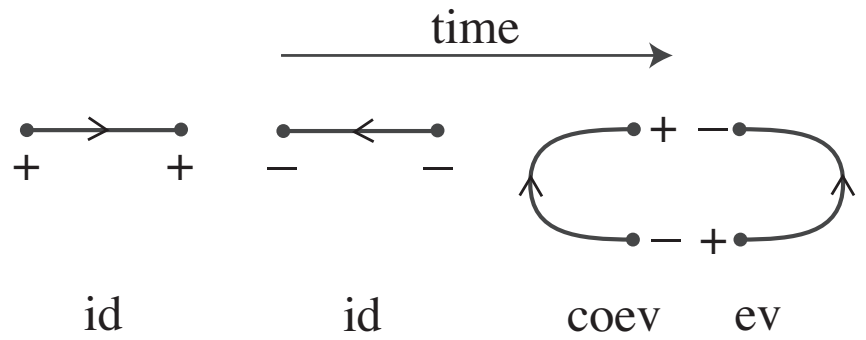

FiguRE 10. Elementary oriented 1-dimensional bordisms 
they are disjoint unions of + and - points. Also, since the empty 0 -manifold $\emptyset^{0}$ is the tensor unit for disjoint union, it maps under the homomorphism $F$ to the tensor unit for complex vector spaces under tensor product, which is the complex line $\mathbb{C}$. Next, consider $F$ evaluated on the bordisms in Figure 10, As $F$ is a homomorphism it sends identities to identities, so the first two bordisms map to $\mathrm{id}_{V_{+}}$and $\mathrm{id}_{V_{-}}$, respectively. The last two bordisms map under $F$ to linear maps

$$
c: \mathbb{C} \longrightarrow \begin{array}{cc}
V_{+} & V_{-} \\
\otimes & e: \otimes \\
V_{-} & V_{+}
\end{array} \longrightarrow \mathbb{C},
$$

where we have written the tensor product vertically to match the figure. The sense in which coevaluation and evaluation give rise to duality is illustrated in Figure 11 . The left figure is the composition of two 1-dimensional bordisms, each with two components. The first maps a single + point to the tensor product (disjoint union) of three points:,,+-+ . The second maps these three points back to the + point. The composition is computed by gluing at the three points in the middle. The result is diffeomorphic to the identity map on the + point. Recall that morphisms in $\operatorname{Bord}_{\langle 0,1\rangle}^{S O}$ are 1-dimensional bordisms up to diffeomorphisms which preserve the boundary identifications. Comparing the first composition in Figure 11 with the first bordism in Figure 10, we see that the composition is the identity. To see the relation to duality we apply the homomorphism $F$. Now the homomorphism property has two consequences: (1) $F$ sends a disjoint union of bordisms to the tensor product of the corresponding linear maps, and (2) $F$ sends a composition of bordisms to the corresponding composition of linear maps. Using these rules we see that $F$ sends the compositions in Figure 11 to compositions of linear maps

$$
\begin{aligned}
& V_{+} \stackrel{\mathrm{id}_{V_{+}}}{\longrightarrow} V_{+} \\
& \begin{array}{lll}
\otimes & \otimes & e
\end{array} \\
& V_{-} \quad \otimes
\end{aligned}
$$

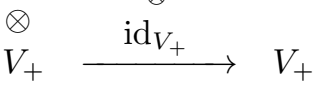

$$
\begin{aligned}
& \begin{array}{cccc}
V_{-} \quad \stackrel{\mathrm{id}_{V_{-}}}{\longrightarrow} & V_{-} & \\
\otimes & \otimes & e \\
c & V_{+} & \otimes \\
& \otimes & \mathrm{id}_{V_{+}} & \\
& V_{-} & V_{-}
\end{array}
\end{aligned}
$$

(Note that we have used the symmetry in the first diagram to exchange the order of the tensor product in the maps $c, e$ from (4.3).)

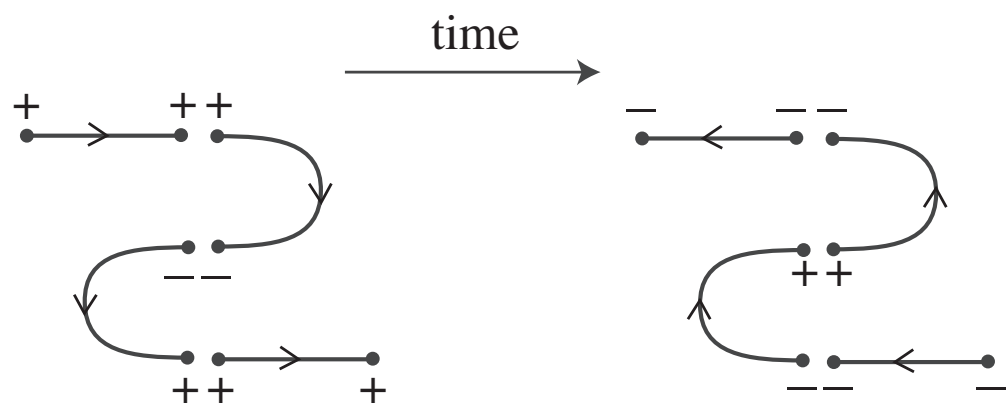

Figure 11. The S-diagrams 
Lemma 4.5. If the compositions (4.4) are identity maps, then $V_{+}, V_{-}$are finite dimensional vector spaces and $e$ is a nondegenerate duality pairing.

Proof. Set $c(1)=\sum_{i=1}^{N} v_{+}^{i} \otimes v_{-}^{i}$ for some $v_{ \pm}^{i} \in V_{ \pm}$and some positive integer $N$. Then the first composition in (4.4) is the map $\xi \mapsto \sum e\left(v_{-}^{i}, \xi\right) v_{+}^{i}$. Since this is the identity map, it follows that $\left\{v_{+}^{i}\right\}_{i=1}^{N}$ spans $V_{+}$, whence $V_{+}$is finite dimensional. The same argument with the second composition proves that $V_{-}$is finite dimensional. If $\xi \in V_{+}$satisfies $e\left(v_{-}, \xi\right)=0$ for all $v_{-} \in V_{-}$, then $\xi=\sum e\left(v_{-}^{i}, \xi\right) v_{+}^{i}=0$. Similarly, using the second composition in (4.4), we deduce that if $\eta \in V_{-}$satisfies $e\left(\eta, v_{+}\right)=0$ for all $v_{+} \in V_{+}$, then $\eta=0$. Hence $e$ is a nondegenerate pairing.

Remark 4.6. A similar argument for a field theory $F:\left(\operatorname{Bord}_{\langle 0,1\rangle}^{S O}, \amalg\right) \longrightarrow(\mathrm{Ab}, \otimes)$ with values in abelian groups proves that $F\left(\mathrm{pt}_{+}\right)$is finitely generated and free.

Lemma 4.5illustrates an important finiteness principle in topological field theories: the vector space attached to an $(n-1)$-manifold in an $n$-dimensional topological field theory with values in Vect $_{\mathbb{C}}$ is finite dimensional. We derived this finiteness from duality: the + point and - point are duals, and that duality is expressed as the existence of coevaluation and evaluation maps. Notice that any vector space $V$ has a dual space, defined algebraically as the space of linear maps $V \rightarrow \mathbb{C}$, which comes with a canonical evaluation map. However, the coevaluation map exists if and only if $V$ is finite dimensional.

This notion of finiteness generalizes to any symmetric monoidal category.

Definition 4.7. Let $\mathcal{C}$ be a symmetric monoidal category and $x \in \mathcal{C}$. Then duality data for $x$ is a triple $\left(x^{\prime}, c, e\right)$ consisting of an object $x^{\prime} \in \mathcal{C}$, a coevaluation $c: 1 \rightarrow$ $x \otimes x^{\prime}$, and an evaluation $e: x^{\prime} \otimes x \rightarrow 1$ such that the compositions

$$
x \stackrel{c \otimes \mathrm{id}_{x}}{\longrightarrow} x \otimes x^{\prime} \otimes x \stackrel{\mathrm{id}_{x} \otimes e}{\longrightarrow} x \quad x^{\prime} \stackrel{\mathrm{id}_{x^{\prime}} \otimes c}{\longrightarrow} x^{\prime} \otimes x \otimes x^{\prime} \stackrel{e \otimes \mathrm{id}_{x^{\prime}}}{\longrightarrow} x^{\prime}
$$

are identity maps. We say $x$ is dualizable if there exists duality data for $x$.

The argument in Lemma 4.5] with the S-diagrams in Figure 11 applies in any $n$-dimensional field theory - take the Cartesian product of the S-diagrams with a fixed $(n-1)$-manifold - which shows that objects in the image of a field theory $F$ are always dualizable. In the next section we define an extension of the notion of a field theory and there is a corresponding extension of dualizability, which we take up in 86 .

At this point we can state and prove a very simple special case of the cobordism hypothesis.

Theorem 4.9. Let $V$ be a finite-dimensional complex vector space. Then there is a homomorphism $F$ as in (4.2) such that $F\left(\mathrm{pt}_{+}\right)=V$.

Proof. If $Y$ is an oriented compact 0-manifold, set

$$
F(Y)=\bigotimes_{y \in Y: y=\mathrm{pt}_{+}} V \otimes \bigotimes_{y \in Y: y=\mathrm{pt}_{-}} V^{*}
$$

Referring to the third and fourth bordisms in Figure 10, define $F(\mathrm{coev})$ as the map $\mathbb{C} \rightarrow V \otimes V^{*}$ which takes $1 \in \mathbb{C}$ to the identity $\operatorname{map}_{V} \in \operatorname{End}(V) \cong V^{*} \otimes V$ and $F(\mathrm{ev})$ as the duality pairing $V^{*} \otimes V \rightarrow \mathbb{C}$. A Morse function on a 1-dimensional bordism decomposes it as a composition of the elementary bordisms coev and ev: a nondegenerate critical point of a real-valued function on a 1-manifold is either a 


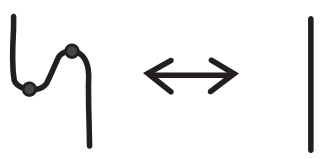

Figure 12. Cerf move in dimension one

local maximum or a local minimum. The only Cerf move (Figure 12) cancels a local maximum against a local minimum, and the proof that this does not change the value of $F$ is the statement that the S-diagrams in Figure 11 map to the identity.

2-dimensional theories. Next, consider a 2-dimensional oriented topological field theory

$$
F:\left(\operatorname{Bord}_{\langle 1,2\rangle}^{S O}, \amalg\right) \longrightarrow\left(\text { Vect }_{\mathbb{C}}, \otimes\right) .
$$

There is only one compact connected oriented 1-manifold up to diffeomorphism: a circle has orientation-reversing diffeomorphisms (reflection). Let $V=F\left(S^{1}\right)$. Elementary 2-dimensional bordisms, as depicted in Figure 13, give extra structure on $V$, namely linear maps

$$
\begin{array}{ll}
m: & V \otimes V \longrightarrow V \\
1: & \mathbb{C} \longrightarrow V \\
\tau: & V \longrightarrow \mathbb{C} .
\end{array}
$$

The multiplication $m$ gives $V$ an algebra structure with respect to which the image of $1 \in \mathbb{C}$ under the linear map 1 is an identity element. The linear map $\tau$ is a trace on $V$.7 Standard arguments with oriented surfaces and their diffeomorphisms prove that $m$ is associative and commutative and that the trace is nondegenerate in the sense that the pairing $v_{1}, v_{2} \mapsto(\tau \circ m)\left(v_{1}, v_{2}\right)$ is a nondegenerate pairing on $V$. For example, the composition of the bordisms labeled $m$ and $\tau$ in Figure 13 is the product of the circle with the bordism labeled ev in Figure 10, then the argument of Lemma 4.5 with the S-diagram proves that the pairing $\tau \circ m$ is nondegenerate. Thus an oriented 2-dimensional topological field theory determines a commutative Frobenius algebra, a commutative algebra with a nondegenerate trace. The converse is also true.

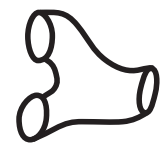

$m$

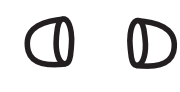

$1 \tau$

FiguRE 13. Some elementary oriented 2-dimensional bordisms

\footnotetext{
${ }^{7}$ Note that the bordism $\tau$ is the time-reversal of 1 . There is also a time-reversal of $m$, which may be expressed as a composition of the maps in (4.12) together with the inverse to the nondegenerate bilinear pairing $\tau \circ \mathrm{m}$.
} 
Theorem 4.13. Let $V$ be a commutative Frobenius algebra. Then there is a homomorphism

$$
F:\left(\operatorname{Bord}_{\langle 1,2\rangle}^{S O}, \amalg\right) \longrightarrow\left(\operatorname{Vect}_{\mathbb{C}}, \otimes\right)
$$

with $F\left(S^{1}\right)=V$.

This is one of the oldest theorems in the subject. In the physics literature the statement dates at least to Dijkgraaf's thesis Di]. There are several proofs in the mathematics literature, for example in $\mathrm{Ab}, \mathrm{Ko}$. The appendix to $\mathrm{MS}$ ] contains a proof of Theorem 4.13 as well as several important variations. As in the proof of Theorem 4.9 we first extend $F$ to all closed oriented 1-manifolds via tensor products. The data (4.12) which defines the Frobenius structure on $V$ tells what to attach to elementary 2-dimensional bordisms arising from critical points of a Morse function of index 1,0,2. It remains to verify that different Morse functions lead to the same linear map. That check, for which we refer to the reader to [MS, uses the basic properties of a commutative Frobenius algebra.

These explicit arguments with Morse functions quickly become tedious and difficult to execute. The situation simplifies for extended field theories (\$5) which are more local. They are the province of the cobordism hypothesis. The cobordism hypothesis is proved using on the one hand more powerful results about spaces of Morse functions and on the other more sophisticated algebra to organize the argument.

One example of a commutative Frobenius algebra is the cohomology algebra $H^{\bullet}(M ; \mathbb{C})$ of a compact oriented $n$-manifold $M$. The trace is pairing with the fundamental class $[M] \in H_{n}(M)$. If there is odd cohomology, then it is commutative in the graded sense because of signs in the commutation rule for cup products. For example, if $M=S^{2}$, then we obtain the truncated polynomial algebra $\mathbb{C}[x] /\left(x^{2}\right)$. The corresponding 2-dimensional topological field theory plays a role in the construction of Khovanov homology for links [Kh, B-N]. If the Frobenius algebra $V$ is semisimple, then we can simultaneously diagonalize the multiplication operators $M_{a}(b)=a b, a, b \in V$ and so find a basis of commuting idempotents $e_{1}, e_{2}, \ldots, e_{n} \in V$ : thus $e_{i} e_{i}=e_{i}$ and $e_{i} e_{j}=0$ if $i \neq j$. The Frobenius algebra is determined up to isomorphism by nonzero complex numbers $\lambda_{1}, \lambda_{2}, \ldots, \lambda_{n}$ defined by $\tau\left(e_{i}\right)=\lambda_{i}$. In this case everything in the field theory $F$ with $F\left(S^{1}\right)=V$ is easily computed in terms of the basis $\left\{e_{i}\right\}$ and the numbers $\lambda_{i}$. For example the two-holed torus in Figure 14 maps to the endomorphism $e_{i} \mapsto \lambda_{i}^{-1} e_{i}$ of $V$ and a closed surface $X_{g}$ of genus $g$ maps to the complex number

$$
F\left(X_{g}\right)=\sum \lambda_{i}^{1-g} .
$$

These computations are made by chopping the surfaces into the elementary bordisms in Figure 13 and their time-reversals.

Let $G$ be a finite group and $A=\operatorname{Map}(G, \mathbb{C})$ the vector space of complex-valued functions on $G$. Then $A$ is an associative algebra under the convolution product

$$
\left(f_{1} * f_{2}\right)(g)=\sum_{g_{1} g_{2}=g} f_{1}\left(g_{1}\right) f_{2}\left(g_{2}\right), \quad g, g_{1}, g_{2} \in G, \quad f_{1}, f_{2}: G \rightarrow \mathbb{C} .
$$

We also define the trace

$$
\tau(f)=\frac{f(e)}{\# G},
$$




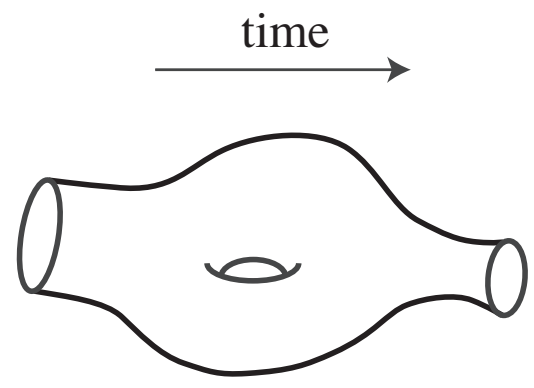

FiguRE 14. Torus with incoming and outgoing boundary circles

where $e \in G$ is the identity element. The product is not commutative if $G$ is not abelian. Let $V$ be the center of $A$, the space of class functions on $G$; it is a commutative Frobenius algebra which can be identified with the complexification $R(G) \otimes \mathbb{C}$ of the representation ring of $G$. Let $F_{G}$ denote the 2-dimensional oriented topological field theory with $F_{G}\left(S^{1}\right)=V$ guaranteed by Theorem 4.13. The complexified representation ring is semisimple. Classical orthogonality formulas of Schur show that the characters $\chi_{i}$ of the irreducible complex representations of $G$ are, up to scale, the commuting idempotents $e_{i}=\left(\chi_{i}(1) / \# G\right) \chi_{i}$. Then we easily compute that $\lambda_{i}=\sum \chi_{i}(1)^{2} / \# G$ and from (4.15) the partition function of a closed connected oriented surface is

$$
F_{G}(X)=\sum_{\substack{\chi \text { irreducible } \\ \text { character of } G}}\left(\frac{\chi(e)}{\# G}\right)^{\text {Euler }(X)},
$$

where $\operatorname{Euler}(X)$ is the Euler characteristic of $X$.

The construction of $F_{G}$ which relies on Theorem 4.13 takes as input the complexified representation ring and uses Morse theory to produce a topological field theory. There is also a direct geometric construction of this simple finite theory. For any manifold $M$ let $\mathscr{F}_{M}$ denote the collection of principal $G$-bundles $P \rightarrow M$. So $P$ is a manifold with a free right $G$-action and quotient $M$. In other terms $P \rightarrow M$ is a covering space which is regular (Galois), but note that $P$ need not be connected. For example, if $M=S^{1}$ and $G=\mathbb{Z} / n \mathbb{Z}$ for some positive integer $n$, then there are $n$ distinct isomorphism classes of principal $G$-bundles over $M$; the connectivity of the total space of a cover depends on the prime factorization of $n$. For any manifold $\mathscr{F}_{M}$ is a category: a morphism $\left(P^{\prime} \rightarrow M\right) \longrightarrow(P \rightarrow M)$ is a smooth map $\varphi: P^{\prime} \rightarrow P$ which commutes with the $G$-action and covers the identity map of $M$. This category is a groupoid since all morphisms are invertible. For $M=$ pt there is only one $G$-bundle up to isomorphism, the trivial bundle $P=G$ with $G$ acting by right multiplication, and the group of automorphisms is $G$ acting by left multiplication on $P$. Figure 15 depicts a groupoid equivalent to $\mathscr{F}_{\text {pt }}$. There is a single object, the set of arrows is $G$, and composition of arrows is given by the group law. For $M=S^{1}$ if we introduce a basepoint $p \in P$ on a $G$-bundle $P \rightarrow S^{1}$, then we can compute the holonomy, or monodromy, around the circle (after choosing an orientation), which is an element of $G$. The bundle with basepoint is rigid: any automorphism which fixes the basepoint is the identity. The group $G$ acts simply transitively on the set of basepoints over a fixed point of $S^{1}$, 


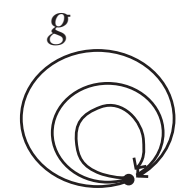

FiguRE 15. G-bundles over pt
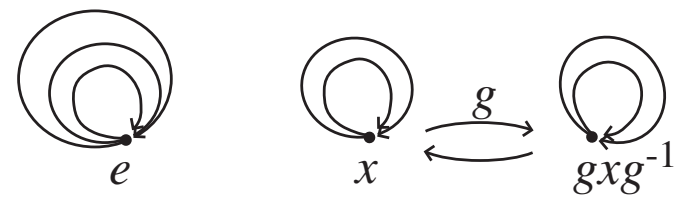

Figure 16. $G$-bundles over $S^{1}$

and it conjugates the holonomy. In this way we see that $\mathscr{F}_{S^{1}}$ is equivalent to the groupoid $G / / G$ of $G$ acting on itself by conjugation. It is depicted in Figure 16. The set of isomorphism classes $\pi_{0}\left(\mathscr{F}_{S^{1}}\right)$ is the set of conjugacy classes in $G$ and the automorphism group $\pi_{1}\left(\mathscr{F}_{S^{1}}, P\right)$ at a $G$-bundle with holonomy $x$ is the centralizer group of $x$ in $G$.

Principal $G$-bundles are local and contravariant. Consider a bordism, as in Figure 1 with the arrow of time pointing to the right. The inclusions of the incoming and outgoing boundary induce restriction maps of bundles, which are homomorphisms of groupoids:

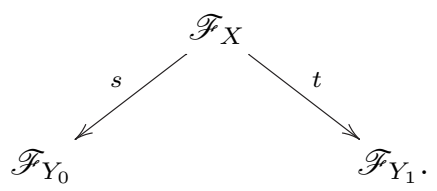

A diagram of the form (4.19) is a correspondence, which is a generalization of a homomorphism from $\mathscr{F}_{Y_{0}}$ to $\mathscr{F}_{Y_{1}}$. Namely, if $s$ is invertible, then $s \times t$ embeds $\mathscr{F}_{X}$ into $\mathscr{F}_{Y_{0}} \times \mathscr{F}_{Y_{1}}$ as the graph of $t \circ s^{-1}$. A composition of bordisms (Figure 3) induces a composition of correspondences

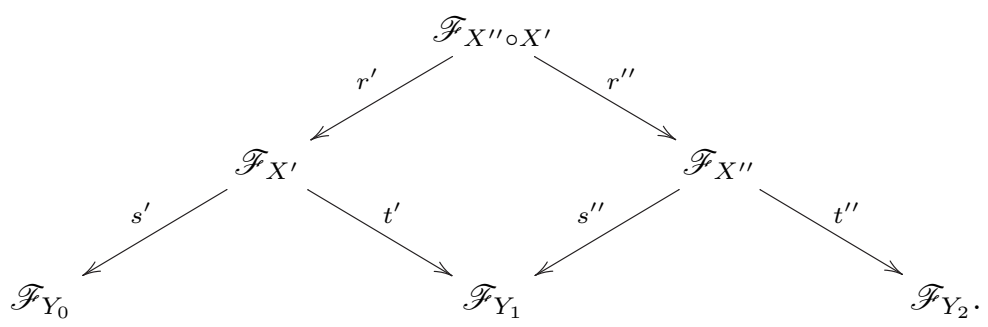

The locality of principal $G$-bundles is hidden in this statement: the groupoid $\mathscr{F}_{X^{\prime \prime} \circ X^{\prime}}$ of $G$-bundles on the composition $X^{\prime \prime} \circ X^{\prime}$ is the fiber product of $t^{\prime}$ and $s^{\prime \prime}$; that is, a $G$-bundle $P \rightarrow X^{\prime \prime} \circ X^{\prime}$ is a triple $\left(P^{\prime}, P^{\prime \prime}, \theta\right)$ consisting of $G$-bundles $P^{\prime} \rightarrow X^{\prime}, P^{\prime \prime} \rightarrow X^{\prime \prime}$, and an isomorphism $\theta:\left.\left.P^{\prime}\right|_{Y_{1}} \rightarrow P^{\prime \prime}\right|_{Y_{1}}$ of their restrictions to $Y_{1}$. 
Correspondence diagrams can often be linearized into honest maps. For the field theory $F_{G}$ we use closed oriented 1-manifolds $Y$ and compact oriented 2-dimensional bordisms $X$. On 1-manifolds we define

$$
F_{G}(Y)=\operatorname{Hom}\left(\mathscr{F}_{Y}, \mathbb{C}\right) .
$$

Here we view $\mathbb{C}$ as a groupoid with only identity morphisms. Then homomorphisms $\mathscr{F}_{Y} \rightarrow \mathbb{C}$ assign complex numbers to objects in $\mathscr{F}_{Y}$ so that the numbers at each end of a morphism are equal. In other words, $\operatorname{Hom}\left(\mathscr{F}_{Y}, \mathbb{C}\right)$ is the vector space of invariant functions on $\mathscr{F}_{Y}$, so it can be identified with $\operatorname{Map}\left(\pi_{0}\left(\mathscr{F}_{Y}\right), \mathbb{C}\right)$, the space of functions on equivalence classes of $G$-bundles. Then to a correspondence (4.19) we define

$$
F_{G}(X)=t_{*} \circ s^{*}: F_{G}\left(Y_{0}\right) \longrightarrow F_{G}\left(Y_{1}\right)
$$

as pullback followed by pushforward. The fibers of $t$ are (equivalent to) groupoids with finitely many objects, each with a finite stabilizer group. The pushforward $t_{*}$ of a function $\phi$ on $\mathscr{F}_{X}$ is the sum

$$
t_{*}(\phi)(y)=\sum_{x} \frac{\phi(x)}{\# \operatorname{Aut}(x)}, \quad y \in \mathscr{F}_{Y_{1}},
$$

over the equivalence classes $x$ in the fiber $t^{-1}(y)$ of the value of $\phi$ divided by the order of the automorphism group. (This formula makes clear that $F_{G}$ may be defined on rational vector spaces.) Key point: The fact that (4.20) is a fiber product implies that the push-pull construction takes compositions of bordisms to compositions of linear maps. In other words, there is an a priori proof that the pushpull construction produces a homomorphism $F_{G}: \operatorname{Bord}_{\langle 1,2\rangle}^{S O} \rightarrow \operatorname{Vect}_{\mathbb{C}}$ of symmetric monoidal categories. The enterprising reader can now compute that $F_{G}\left(S^{1}\right)$ is the vector space of central functions on $G$, and that the basic bordisms in Figure 13 map to the convolution product, the character of the identity representation, and the trace (4.17).

Now suppose $X$ is a closed oriented 2-manifold. It is interpreted as a bordism $X: \emptyset^{1} \rightarrow \emptyset^{1}$. In grand Bourbaki style the groupoid of $G$-bundles $\mathscr{F}_{\emptyset^{1}}$ has a single object with only the identity morphism. (After all, $\mathscr{F}$ maps disjoint unions to Cartesian products, and $\emptyset^{1}$ is the tensor unit for disjoint union.) In this case (4.22) specializes to the sum of the constant function 1 over $\mathscr{F}_{X}$ : it counts (with automorphisms) the $G$-bundles over $X$. If $X$ is connected, then that count of bundles is

$$
F_{G}(X)=\frac{\# \operatorname{Hom}\left(\pi_{1}(X, x), G\right)}{\# G} ;
$$

the numerator counts $G$-bundles with a basepoint over $x$ and the group $G$ acts simply transitively on the basepoints.

Theorem 4.25. Let $X$ be a compact oriented connected 2-manifold and $G$ a finite group. Then

$$
\# \operatorname{Hom}\left(\pi_{1}(X, x), G\right)=(\# G) \sum_{\begin{array}{c}
\chi \text { irreducible } \\
\text { character of } G
\end{array}}\left(\frac{\chi(1)}{\# G}\right)^{\operatorname{Euler}(X)},
$$

where $\operatorname{Euler}(X)$ is the Euler characteristic of $X$. 
The theorem follows immediately by comparing (4.24) and (4.18). It was known to Frobenius and Schur from the character theory of finite groups, with no quantum fields in sight. The proof given here is representative of how topological field theory is used in more complicated situations. The invariant on the left-hand side of (4.26), initially defined for closed 2-manifolds, is extended to an invariant for compact 2-manifolds with boundary which obeys a gluing law. So it is computed by chopping $X$ into elementary pieces (as in Figure 13 together with the timereversal of $m$ ).

Remark 4.27. The appearance of the Euler characteristic in (4.26) suggests an extension of $F_{G}$ which includes 0 -manifolds. They would appear as corners of 2manifolds and boundaries of 1-manifolds. Then in a triangulation of $X$, the count of vertices, edges, and triangles in the triangulation should combine to give the Euler characteristic Euler $(X)$ and a new proof of (4.26). In such an extended field theory we have more locality, so more decompositions and hence more computational flexibility. We take up extended theories in $\$ 5$ and pursue this idea in Example 5.7.

Remark 4.28. There is a variation on (4.22) in which $\mathscr{F}_{X}$ in (4.19) carries an integral kernel. In that case the pull-push formula (4.22) is modified to pull-multiply-push. The integral kernel must be local in that it multiplies in the fiber product (4.20). In this 2-dimensional theory we can obtain such an integral kernel by starting with a cocycle for a class in the group cohomology $H^{2}(G ; \mathbb{C} / \mathbb{Z})$.

The theory $F_{G}$ was introduced by Dijkgraaf and Witten [DW]. See [FQ and $[\mathrm{F}]$ for more detail about defining $F_{G}$ by counting principal $G$-bundles. The lecture notes Q contain elaborations and many more examples.

The push-pull construction is a finite version of the Feynman functional integral in quantum field theory. The groupoid $\mathscr{F}_{M}$ consists of gauge fields for a finite group $G$; if $G$ is a Lie group, then gauge fields form the groupoid of $G$-connections on $M$. The integral kernel described in Remark 4.28 is the exponential of the classical action of the field theory. The pushforward $t_{*}$ is the Feynman integral or functional integral or path integral over the space of fields (with fixed boundary condition). In almost all physically interesting examples the space, or stack, of fields is not finite, but rather is infinite dimensional. One way to define pushforward $t_{*}$ on functions is via integration theory, which of course requires a measure on the space of fields. (There are alternatives, at least for some topological theories; see [FHT] for one example.) Furthermore, the measures must be consistent with the fiber product (4.20) under composition of bordisms. Such measures have not been constructed rigorously in most examples of physical interest. The example of finite gauge theories, while it nicely illustrates many topological and algebraic aspects, misses completely the central analytical issues in quantum field theory.

\section{5. $n$-CATEGORIES AND EXTENDED TOPOLOGICAL QUANTUM FIELD THEORY}

In this section we extend the definition of an $n$-dimensional topological field theories in two directions:

(i) to invariants of manifolds of all dimensions $\leq n$; and

(ii) to invariants of families of manifolds.

These extensions go beyond what was traditionally done in quantum field theory. 


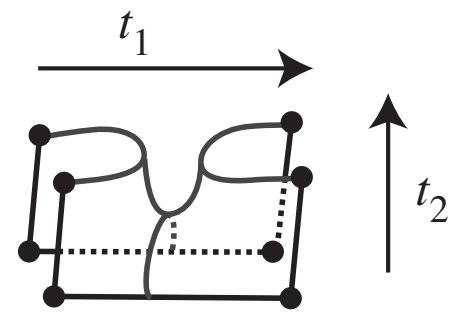

FiguRE 17. Two-time evolution of two points

Standard topological field theories, as in Definition 2.6, are local in that invariants of $n$-manifolds are computed by cutting along closed codimension 1 submanifolds. We saw after Theorem 4.25, and even in the description of classical Morse theory (Remark 2.8), that it is desirable to go further and cut along codimension 2 submanifolds as well, so we have $n$-manifolds with corners. Once we take that plunge, we may as well continue cutting in higher and higher codimension until we are cutting along 0 -manifolds. In other words, we end up considering $n$-manifolds with corners of all codimensions. The local model for the maximal corner is a corner in real affine space: $\left\{\left(x^{1}, x^{2}, \ldots, x^{n}\right) \in \mathbb{A}^{n}: x^{i} \geq 0\right\}$ near $(0,0, \ldots, 0)$.

In a bottom-up view, rather than a top-down view, we build higher-dimensional manifolds by time evolution of lower-dimensional manifolds. This is illustrated in Figure 10 by the time evolution of 0 -manifolds to produce 1 -manifolds. Now we evolve again, introducing a second time as in Figure 17. Let $t_{1}, t_{2} \in[0,1]$ denote the times, so the space of times is the square $[0,1] \times[0,1]$. At each of the four corners $t_{1}, t_{2} \in\{0,1\}$ lies the 0 -manifold $Y$ consisting of two points. At time $t_{2}=0$ they evolve in $t_{1}$ via the identity bordism, whereas at time $t_{2}=1$ they evolve as the evaluation followed by the coevaluation. (These 1-dimensional bordisms are pictured in Figure 10). The evolution in $t_{2}$ is a 2-dimensional bordism $W$ between these two 1-dimensional bordisms $X_{0}, X_{1}$. As a manifold it is a 2-dimensional manifold with corners, but as a bordism we remember the time evolutions. Morally, as in 92 it is only the arrows of time which matter - and these only near the boundaries and corners - but it is convenient both heuristically and technically to think in terms of actual time functions. An algebraic representation of this two-time evolution is:

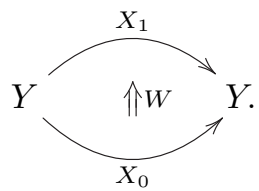

The algebraic structure which includes (5.1) is a 2-category. In addition to objects $x, y$ and morphisms $f, g: x \rightarrow y$ mapping between them, there are now 2morphisms $\eta: f \Rightarrow g$ which map between morphisms. For clarity "morphisms" are now termed "1-morphisms". In the 2-category Bord ${ }_{\langle 0,1,2\rangle}$ the objects are compact 0-manifolds, the 1-morphisms are 1-dimensional bordisms, and the 2-morphisms are 2-dimensional bordisms. A 2-category has two associative composition laws, easily seen pictorially in Bord $\alpha_{\langle 0,1,2\rangle}$. Namely, we can compose horizontally in the first time $t_{1}$ or vertically in the second time $t_{2}$. Disjoint union is an extra algebraic 
structure - still called a symmetric monoidal structure - and the empty manifolds are identity elements for disjoint union. So, for example, a closed 2-manifold $W$ is interpreted as a 2-morphism $W: \emptyset^{1} \Rightarrow \emptyset^{1}$ in $\operatorname{Bord}_{\langle 0,1,2\rangle}$. For now we leave unspecified what sort of extra topological data (orientation, framing, ... ) we assume present.

The saddle in Figure 17 is the elementary bordism in Morse theory depicted in Figure 4. In other words, it is the 2-manifold $D^{1} \times D^{1}$ which implements the surgery beginning with $S^{0} \times D^{1}$ and ending with $D^{1} \times S^{0}$. Here $D^{1}$ is the standard closed 1-ball. The general surgery

$$
D^{p} \times D^{q}: S^{p-1} \times D^{q} \longrightarrow D^{p} \times S^{q-1},
$$

can be written algebraically in a diagram similar to (5.1) with $Y=S^{p-1} \times S^{q-1}$. Morse theory tells that a manifold has a handlebody decomposition into elementary bordisms (5.2). We might conclude that 2-categories go far enough, and that nothing is to be gained by chopping further. We could, after all, make a 2-category whose objects are closed $(n-2)$-manifolds and with 1-morphisms and 2-morphisms their time evolutions. But the structure simplifies if we do not stop there and rather go all the way down to points.

Therefore, to study manifolds of dimension $\leq n$, or equivalently to study topological field theories of dimension $n$, we are led to the $n$-category $\operatorname{Bord}_{\langle 0 \cdots n\rangle}$ whose objects are compact 0 -manifolds and whose $k$-morphisms $(1 \leq k \leq n)$ are $k$-time evolutions of objects. There are $k$ composition laws for $k$-morphisms, and they satisfy various compatibilities. Disjoint union gives a symmetric monoidal structure. It is a complicated combinatorial problem to track all of this data. The relevance of higher categories to topological field theory was understood in the early 1990s, but at that time rigorous foundations were not available. In the intervening years several approaches and definitions have been advanced. We will not attempt a formal definition here, but refer the reader to [BD, L1] for more detailed exposition and references.

The $n$-category $\operatorname{Bord}_{\langle 0 \cdots n\rangle}$ is the first extension we envisioned at the beginning of this section. The second is to families of manifolds. It turns out that this can be encoded by extending the $n$-category $\operatorname{Bord}_{\langle 0 \cdots n\rangle}$ higher up: we adjoin $(n+1)$-morphisms, $(n+2)$-morphisms, etc. Namely, if $W_{0}, W_{1}$ are $n$-dimensional bordisms, we define an $(n+1)$-morphism $\varphi: W_{0} \rightarrow W_{1}$ to be a diffeomorphism which preserves all of the "boundary data". An $n$-morphism is a map between two $(n-1)$-morphisms, each of which is a map between two $(n-2)$-morphisms, and on down. The diffeomorphism $\varphi$ must preserve the implicit identifications. In terms of the $n$-time evolution, $\varphi$ must be compatible with the data at each of the $2^{n}$ extreme times $t_{i} \in\{0,1\}$. Since $\varphi$ is a diffeomorphism, it is invertible. We continue and define an $(n+2)$-morphism $\varphi_{0} \rightarrow \varphi_{1}$ to be an isotopy between the diffeomorphisms $\varphi_{0}$ and $\varphi_{1}$, again preserving the boundary data. Isotopies are also invertible, up to a higher isotopy. Continuing in this way we have $k$-morphisms for all $k$, so an $\infty$-category. But it has the property that every $k$-morphism for $k>n$ is invertible.

Definition 5.3. Let $n \in \mathbb{Z}^{>0}$. An $(\infty, n)$-category is an $\infty$-category in which every $k$-morphism is invertible for $k>n$. 
"Definition" is not really appropriate as we have not defined $\infty$-categories! There are complete definitions for $(\infty, n)$-categories, in fact several $[\mathrm{Ba}, \mathrm{R}, \mathrm{Be}]$ with others on the way, and also a study [BS] of all homotopy theories of $(\infty, n)$-categories.

Remark 5.4. A higher category in which every morphism is invertible-i.e., an $(\infty, 0)$-category - is a combinatorial model for a space. Since every morphism is invertible, this is also called an $\infty$-groupoid. So whereas an $n$-category has sets of $n$-morphisms, an $(\infty, n)$-category has spaces of $n$-morphisms. An $n$-category may be extended to a discrete $(\infty, n)$-category in which all $k$-morphisms for $k>n$ are identity maps.

Definition 5.5. Bord $_{n}$ is the $(\infty, n)$-category whose objects are compact 0 -manifolds, $k$-morphisms for $1 \leq k \leq n$ are $k$-time evolutions of objects, and $k$-morphisms for $k>n$ are $(k-n)$-fold iterated isotopies of diffeomorphisms. It is symmetric monoidal under disjoint union.

Again this is only a descriptive definition.

The manifolds in Bord $_{n}$ typically carry extra data. For example, there is an $(\infty, n)$-category $\operatorname{Bord}_{n}^{S O}$ of oriented bordisms. There is also a bordism category of bordisms with tangential framing, but in an unstable 8 sense. Namely, an $n$-framing on a $k$-bordism $W$ in $\operatorname{Bord}_{n}^{\mathrm{fr}}$ is a trivialization of $T W \oplus(n-k)$, where $(n-k)$ is the trivial bundle of the indicated rank. The $(\infty, n)$-category of unoriented manifolds is denoted $\operatorname{Bord}_{n}^{O}$. We use " $\operatorname{Bord}_{n}$ " generically to denote any of these and many other similar possibilities.

Analogous to Definition 2.6 we consider representations of $\operatorname{Bord}_{n}$. We allow an arbitrary codomain.

Definition 5.6. Let $\mathcal{C}$ be a symmetric monoidal $(\infty, n)$-category. An extended topological field theory with values in $\mathcal{C}$ is a homomorphism $F: \operatorname{Bord}_{n} \rightarrow \mathcal{C}$.

The homomorphism property means that $F$ respects the $n$ composition laws as well as the symmetric monoidal structures. The cobordism hypothesis, which we take up in the next section, determines the space of homomorphisms $F$ in terms of $\mathcal{C}$.

For the remainder of this section we indicate some examples which illuminate the idea of an extended field theory and the flexibility of Definition 5.6.

Example 5.7. Let $G$ be a finite group. Recall from 4 the 2-dimensional topological field theory $F_{G}$ : $\operatorname{Bord}_{\langle 1,2\rangle}^{S O} \rightarrow$ Vect $_{\mathbb{C}}$. In (4.16) we introduced the algebra $A=\operatorname{Map}(G, \mathbb{C})$ of functions on $G$ under convolution, but only its center made an appearance in $F_{G}$ as $F_{G}\left(S^{1}\right)$. There is an extended field theory $\widehat{F}_{G}$ of $0-, 1-$, and 2-manifolds which has $\widehat{F}_{G}\left(\mathrm{pt}_{+}\right)=A$. The codomain $(\infty, 2)$-category $\mathcal{C}$ of any extension has the property that the $(\infty, 1)$-category $\operatorname{Hom}_{\mathcal{C}}(1,1)$ of endomorphisms of the tensor unit 1 is identified with Vect $\mathbb{C}$. In fact, Vect $\mathbb{C}$ is discrete: objects are complex vector spaces, 1-morphisms are linear maps, and there are no nonidentity higher morphisms. So we might hope that $\mathcal{C}$ is also discrete, an ordinary 2-category. Furthermore, if $\widehat{F}_{G}\left(\mathrm{pt}_{+}\right)$is to be $A$, then objects of $\mathcal{C}$ are algebras. Thus let $\mathcal{C}=A l g_{\mathbb{C}}$ be the 2-category whose objects are complex algebras. If $A_{0}, A_{1} \in A l g_{\mathbb{C}}$, then we define a 1-morphism $B: A_{0} \rightarrow A_{1}$ to be an $\left(A_{1}, A_{0}\right)$-bimodule $B$, a complex vector

\footnotetext{
${ }^{8}$ Framings on manifolds used to define framed bordism groups - isomorphic by the PontrjaginThom construction to stable homotopy groups of spheres-are stable framings of the normal bundle.
} 
space $B$ with a left action of $A_{1}$ and a right action of $A_{0}$. Composition is by tensor product over algebras: if $B: A_{0} \rightarrow A_{1}$ and $B^{\prime}: A_{1} \rightarrow A_{2}$, then $B^{\prime} \circ B: A_{0} \rightarrow A_{2}$ is the $\left(A_{2}, A_{0}\right)$-bimodule $B^{\prime} \otimes_{A_{1}} B$. The symmetric monoidal structure is given by a tensor product over $\mathbb{C}$. The algebra $\mathbb{C}$ is the tensor unit 1 and $\operatorname{Hom}_{A l g_{\mathbb{C}}}(1,1)$ is the collection of $(\mathbb{C}, \mathbb{C})$-bimodules, which is canonically Vect $\mathbb{C}$, as desired. A 2-morphism between bimodules is a linear map which intertwines the algebra actions. To put this construction in context, we observe that an isomorphism in the 2-category $A l g_{\mathbb{C}}$ of algebras is a Morita equivalence of algebras.

We pause to remark that we have climbed to the next categorical level-from 1categories to 2-categories - by endowing objects in a 1-category with an associative unital composition law. Complex vector spaces form a 1-category, whereas complex vector spaces which are algebras form a 2-category. This is an important general idea, which can be implemented at all categorical levels and also can be iterated. For example, if we consider complex vector spaces with two composition laws, we obtain a 3-category (of commutative algebras). We will meet more examples below. We can embed $A g_{\mathbb{C}}$ into the more familiar 2-category of $\mathbb{C}$-linear categories $\mathrm{Cat}_{\mathbb{C}}$ : an algebra $A$ maps to the linear category of left $A$-modules. It is usually easier to scale categorical heights by looking at "algebra objects" in an existing category, rather than by introducing new and more elaborate constructs.

Returning to

$$
\widehat{F}_{G}: \operatorname{Bord}_{2}^{S O} \longrightarrow A l g_{\mathbb{C}}
$$

once we posit $\widehat{F}_{G}\left(\mathrm{pt}_{+}\right)=A=\operatorname{Map}(G, \mathbb{C})$, we can compute $\widehat{F}_{G}\left(S^{1}\right)$ as follows. We know that $\widehat{F}_{G}\left(\mathrm{pt}_{-}\right)$is the dual to $\widehat{F}_{G}\left(\mathrm{pt}_{+}\right)$, since $\mathrm{pt}_{+}$and $\mathrm{pt}$ - are dual in Bord ${ }_{2}^{S O}$, and it turns out that the dual algebra is the opposite algebra $A^{\circ}$. The coevaluation in Figure 10 is the left $\left(A \otimes A^{o}\right)$-module $A$, and the evaluation is the right $\left(A^{o} \otimes A\right)$ module $A$. After permuting the two boundary points of the evaluation, we compose coevaluation and evaluation to compute

$$
\widehat{F}_{G}\left(S^{1}\right)=A \otimes_{A \otimes A^{\circ}} A .
$$

This tensor product is the Hochschild homology of the algebra $A$. We can easily compute it explicitly. Tensoring over $A$ gives the tensor product $A \otimes_{A} A$ of the right $A$-module $A$ with the left $A$-module $A$, which is canonically $A$ by multiplication. Then the $A^{o}$-action is by left and right multiplication, so letting $[A, A] \subset A$ denote the subspace spanned by elements of the form $a_{1} a_{2}-a_{2} a_{1}, a_{1}, a_{2} \in A$, we conclude $\widehat{F}_{G}\left(S^{1}\right)=A /[A, A]$. This is not the center of $A$, which is what we expect from the text after (4.23). To identify the vector space $A /[A, A]$ with the center of $A$ we need one more piece of data, a nondegenerate trace $\tau: A \rightarrow \mathbb{C}$ on $A$. Nondegeneracy means that $a_{1}, a_{2} \mapsto \tau\left(a_{1} a_{2}\right)$ is a nondegenerate pairing, and then we identify the quotient $A /[A, A]$ with the orthogonal subspace $[A, A]^{\perp} \subset A$, which is easily identified with the center of $A$. The pair $(A, \tau)$ is a Frobenius algebra. For $A=\operatorname{Map}(G, \mathbb{C})$ we use the trace (4.17).

The cobordism hypothesis, stated for framed manifolds in Theorem 1.2, asserts that $\widehat{F}_{G}$ is determined by its value on $\mathrm{pt}_{+}$. This is true here, but "value on $\mathrm{pt}_{+}$" must be interpreted as the pair $(A, \tau)$. The extra datum $\tau$ is necessary as $\widehat{F}_{G}$ is an oriented theory, not simply a framed theory; see Theorem 6.11 and Example 6.13. 
In $\$ 4$ we described an approach to the nonextended theory $F_{G}$ using a finite version of the path integral in physics, which amounts to counting principal $G$ bundles. The finite path integral extends to give an a priori construction of $\widehat{F}_{G}$ in which $\widehat{F}_{G}\left(\mathrm{pt}_{+}\right)=A$ is the result of a computation; see [F, FHLT for detail.

Example 5.10. Historically, 3-dimensional Chern-Simons theory W3 was the example which most pointed the way towards extended topological field theories. The approach of Reshetikhin-Turaev RT1, RT2 to the resulting invariants of 3manifolds and links begins with a quantum group, in the form of a complex linear category with extra structure, a modular tensor category [MSei]. By contrast, Witten begins with the Chern-Simons functional and uses the path integral. The relationship between the approaches, worked out in $[\mathrm{F}$. for finite gauge groups, is that Chern-Simons is a (partially) extended theory of 1-, 2-, and 3-manifolds whose value on $S^{1}$ is the modular tensor category. A complete construction of this 1-2-3 theory beginning from quantum group data was given in $\mathrm{Tu}$; see also $\mathrm{Wa}$. There is current work, for example $[\mathrm{BDH}]$, to construct a fully extended 0-1-2-3 theory.

Example 5.11. The previous two examples are discrete: there are no interesting invariants for families of manifolds beyond those for single manifolds. That an extension of Definition 2.6 to families would be fruitful emerged in the 1990s from 2dimensional field theories. Segal promoted the idea of a cochain-valued topological field theory [Se3], and there were several mathematical works which pointed towards invariants for families of manifolds; a quirky sample is $[\mathrm{LZ}, \mathrm{G}, \mathrm{KM}, \mathrm{BC}$. The most definitive work in this direction is by Kevin Costello $\mathrm{Co}$, who constructed a theory of open-closed topological 2-dimensional field theories in families from Calabi-Yau categories. These are closely related to fully extended 2-dimensional theories; see [L1, §4.2].

Example 5.12. Another motivating example for the cobordism hypothesis which includes invariants for families of manifolds is string topology, which defines invariants of compact manifolds using its loop space and Riemann surfaces. It was introduced by Chas and Sullivan [CS, and there is a large literature which follows. See [L1, §4.2] for the relation with the cobordism hypothesis.

\section{The COBORDISM HYPOTHESIS}

Recall from $\$ 4$ that objects in the image of a nonextended topological field theory obey a finiteness condition, expressed in categorical terms by dualizability (Definition 4.7). There is an analogous finiteness condition called adjointability for $k$-morphisms, $1 \leq k \leq n-1$, in an extended $n$-dimensional field theory. We give the definition for 1-morphisms, which specializes to the traditional notion of adjoint functors in category theory $\mathrm{Ka}$. for the 2-category of categories.

Definition 6.1. Let $\mathcal{C}$ be a 2-category; $x, y \in \mathcal{C}$ objects in $\mathcal{C}$; and suppose $f: x \rightarrow y$, $g: y \rightarrow x$ are 1-morphisms. Then $f$ is a left adjoint to $g$ if there exist 2-morphisms $u: \operatorname{id}_{x} \Rightarrow g \circ f$ and $c: f \circ g \Rightarrow \operatorname{id}_{y}$ such that the compositions

$$
f=f \circ \operatorname{id}_{x} \stackrel{\operatorname{id} \times u}{\Longrightarrow} f \circ g \circ f \stackrel{c \times \operatorname{id}}{\Longrightarrow} \operatorname{id}_{y} \circ f=f
$$

and

$$
g=\mathrm{id}_{x} \circ g \stackrel{u \times \mathrm{id}}{\Longrightarrow} g \circ f \circ g \stackrel{\mathrm{id} \times c}{\Longrightarrow} g \circ \mathrm{id}_{y}=g
$$

are identity 2-morphisms. 
We then say that $g$ is a right adjoint to $f$, and $u, c$ are the unit and counit of an adjunction. The compositions (6.2) and (6.3) are the 2-morphism version of the S-diagram compositions (4.8). The corresponding definition for $(\infty, n)$-categories and higher morphisms is similar, but the compositions are only the identity maps up to higher morphisms, or equivalently are identity maps in a homotopy category which remembers higher morphisms only up to equivalence. Invertible maps have adjoints - the inverse is an adjoint - so adjointability is weaker than invertibility.

Remark 6.4. If an $n$-morphism in an $n$-category, or $(\infty, n)$-category, is adjointable, then it is invertible. This follows since the unit and counit of an adjunction, which are $(n+1)$-morphisms, are invertible.

Let $\mathcal{C}$ be a symmetric monoidal $(\infty, n)$-category and $F:$ Bord $_{n} \rightarrow \mathcal{C}$ an extended field theory. Then just as $F(\mathrm{pt})$ is dualizable, so too is $F(W)$ adjointable for every $k$-dimensional bordism $W$ with $1 \leq k \leq n-1$. This is an extended finiteness condition satisfied by an extended topological field theory. We extract from $\mathcal{C}$ all objects which have duals, and whose duality data have adjoints, which in turn have adjoints, etc.

Lemma 6.5 ([L1, §2.3]). Let $\mathcal{C}$ be a symmetric monoidal $(\infty, n)$-category. There is an $(\infty, n)$-category $\mathcal{C}^{\mathrm{fd}}$ and a homomorphism $i: \mathcal{C}^{\mathrm{fd}} \rightarrow \mathcal{C}$ so that

(i) every object in $\mathcal{C}^{\mathrm{fd}}$ is dualizable and every $k$-morphism, $1 \leq k \leq n-1$, is adjointable, and

(ii) $i: \mathcal{C}^{\mathrm{fd}} \rightarrow \mathcal{C}$ is universal with respect to (i).

Here "fd" stands either for fully dualizable or finite dimensional. An $(\infty, n)$ category which satisfies (i) is said to "have duals", as in the statement of Theorem 1.1. The finiteness condition on a topological field theory $F: \operatorname{Bord}_{n} \rightarrow \mathcal{C}$ may be summarized by the following diagram.

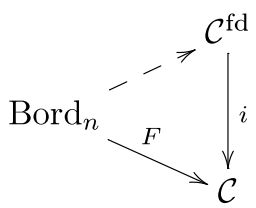

In other words, $F$ factors through $\mathcal{C}^{\mathrm{fd}}$.

Extended topological field theories $F: \operatorname{Bord}_{n} \rightarrow \mathcal{C}$ are the objects of an $(\infty, n)$ category we denote $\operatorname{Hom}\left(\operatorname{Bord}_{n}, \mathcal{C}\right)$. A 1-morphism $\eta: F_{0} \rightarrow F_{1}$ between two homomorphisms assigns a $(k+1)$-dimensional morphism $\eta(W): F_{0}(W) \rightarrow F_{1}(W)$ to each $k$-dimensional bordism $W$. The fact that adjointable $n$-morphisms are invertible (Remark 6.4) implies, after some argument, that any 1-morphism $\eta$ is in fact an isomorphism. The same applies to higher morphisms. It follows that $\operatorname{Hom}\left(\operatorname{Bord}_{n}, \mathcal{C}\right)$ is in fact an $(\infty, 0)$-category - all morphisms are invertible - so according to Remark 5.4 can be viewed as a space. In other words, the collection of extended topological field theories with values in $\mathcal{C}$ is a space.

The cobordism hypothesis identifies the space $\operatorname{Hom}\left(\operatorname{Bord}_{n}, \mathcal{C}\right)$ with a space constructed directly from $\mathcal{C}$ by combining Lemma 6.5 with another universal construction.

Lemma 6.7 ([L1, §2.4]). Let $\mathcal{D}$ be an $(\infty, n)$-category. There is an $\infty$-groupoid $\mathcal{D}^{\sim}$ and a homomorphism $j: \mathcal{D}^{\sim} \rightarrow \mathcal{D}$ so that 
(i) every $k$-morphism, $k>0$, in $\mathcal{D}^{\sim}$ is invertible, and

(ii) $j: \mathcal{D}^{\sim} \rightarrow \mathcal{D}$ is universal with respect to (i).

The $\infty$-groupoid $\mathcal{D}^{\sim}$, which is an $\infty$-category in which every morphism is invertible, may be constructed from $\mathcal{D}$ by removing all noninvertible morphisms.

Finally, we can state a precise version of the cobordism hypothesis, first for $n$-framed manifolds.

Theorem 6.8 (Cobordism hypothesis: framed version). Let $\mathcal{C}$ be a symmetric monoidal $(\infty, n)$-category. Then the map

$$
\begin{aligned}
\operatorname{Hom}\left(\operatorname{Bord}_{n}^{\mathrm{fr}}, \mathcal{C}\right) & \longrightarrow\left(\mathcal{C}^{\mathrm{fd}}\right)^{\sim} \\
F & \longmapsto F\left(\mathrm{pt}_{+}\right)
\end{aligned}
$$

is a homotopy equivalence of spaces.

At this point the reader should refer back to the heuristic versions stated in $₫ 1$ as well as the discrete 1-dimensional version in Theorem 4.9. In particular, the cobordism hypothesis is a theorem about smooth manifolds and their diffeomorphism groups, which is reflected by the method of proof.

Suppose $W$ is a bordism of dimension $k \leq n$ which is $n$-framed. Recall that the $n$-framing is an isomorphism $(n) \rightarrow(n-k) \oplus T W$, where $(j)$ denotes the trivial real vector bundle of rank $j$ over $W$. The orthogonal group $O(n)$ acts on framings by precomposition with constant orthogonal maps $(n) \rightarrow(n)$. This induces an action of $O(n)$ on the space $\operatorname{Hom}\left(\operatorname{Bord}_{n}^{\mathrm{fr}}, \mathcal{C}\right)$.

Corollary 6.10. There is a canonical action of the orthogonal group $O(n)$ on the space $\left(\mathcal{C}^{\mathrm{fd}}\right) \sim$.

Let $G$ be a Lie group equipped with a homomorphism $\rho: G \rightarrow O(n)$. A $G$ structure on a bordism $W$ is a reduction of structure group of its tangent bundle to $G$ along $\rho$. More precisely, choose a Riemannian metric on $W$ (this is a contractible choice). Then a $G$-structure is a principal $G$-bundle $P \rightarrow W$ together with an isomorphism of the associated $G$-bundle $\rho(P)$ with the bundle of orthonormal frames of $(n-k) \oplus T W$. For example, for $G=\{e\}$, a $G$-structure is an $n$-framing, and for $G=S O(n)$, it is an orientation. There is a bordism category $\operatorname{Bord}_{n}^{G}$ of manifolds with $G$-structure.

Theorem 6.11 (Cobordism hypothesis: $G$-structure version). The map

$$
\begin{aligned}
\operatorname{Hom}\left(\operatorname{Bord}_{n}^{G}, \mathcal{C}\right) & \longrightarrow\left(\left(\mathcal{C}^{\mathrm{fd}}\right)^{\sim}\right)^{h G} \\
F & \longmapsto F\left(\mathrm{pt}_{+}\right)
\end{aligned}
$$

is a homotopy equivalence between the space of extended topological field theories on $G$-manifolds and the homotopy fixed point space of the $G$-action on $\left(\mathcal{C}^{\mathrm{fd}}\right)^{\sim}$.

Here $G$ acts through the homomorphism $\rho: G \rightarrow O(n)$ and the $O(n)$-action given in Corollary 6.10.

Example 6.13. For $n=2$ an oriented 2-dimensional theory is determined by the value on $\mathrm{pt}_{+}$, but in the fixed point space. Consider $\mathcal{C}=A l g_{\mathbb{C}}$, as in Example 5.7

\footnotetext{
${ }^{9}$ It is perhaps more natural to use the full general linear group $G L(n ; \mathbb{R})$, but all of the topological information is carried by the maximal compact subgroup $O(n) \subset G L(n ; \mathbb{R})$.
} 
First, the 2-category $A l g_{\mathbb{C}}^{\mathrm{fd}}$ of fully dualizable complex algebras has objects finitedimensional semisimple algebras, i.e., finite products of matrix complex algebras. (A proof may be found in [Da, §3.2].) A point in the homotopy fixed point space of the $S O(2)$-action includes extra data - in this case being a fixed point is not a condition - and the extra data here is the nondegenerate trace $\tau$ discussed in Example 5.7, see [FHLT, Example 2.8] for detail.

We are not going to attempt to summarize the proof sketched in [L1] in any detail. Rather, we give a very rough intuition for why the cobordism hypothesis might be true. Our exposition in $\$ 4$, in particular the proof of Theorem 4.9, emphasizes the role of Morse theory. The existence of Morse functions allows the decomposition of a bordism into a composition of elementary bordisms (5.2). These elementary bordisms encode the evaluations and coevaluations, or units and counits, of duality and adjointness data. That is clear in the proof of Theorem 4.9. As another example, Figure 17 may be read as a counit for the adjunction between the two 1 -morphisms coev, ev in Figure10. So if $x \in \mathcal{C}$ is fully dualizable, a choice of duality data - duals and adjoints all the way up - defines $F$ on elementary bordisms. As arbitrary bordisms are compositions of elementary bordisms, $F$ can be extended to arbitrary bordisms. In other words a Morse function gives, in principle, a way to evaluate $F(W)$ for every bordism $W$. The issue is whether $F(W)$ is well defined. The duality data involves choices, and we must be sure that those choices can be made coherently. This is expressed via contractibility statements. The first is that the space of duality data for a dualizable object $x$ is contractible. The second generalizes the connectivity statement at the heart of Cerf theory [C]. Lurie uses a higher connectivity theorem of Kiyoshi Igusa [I] for the space of generalized framed Morse functions. Such functions relax the nondegeneracy condition at a critical point to allow a single degeneracy, as in (2.9), and also include a framing of the negative definite subspace at a critical point. Igusa proves that on a $k$-dimensional manifold, this space is $k$-connected 10 These contractibility statements are central to the proof, but it is a highly nontrivial problem to organize the higher categorical data to apply these theorems. The solution to that problem, described in detail in [L1], is equally central to the proof.

\section{IMPLiCATIONS, EXTENSIONS, AND APPLICATIONS}

Some brief vignettes illustrate the scope of the extended topological field theory and the cobordism hypothesis.

Invertible theories and Madsen-Tillmann spectra. Recall from Lemma 6.7 that any $(\infty, n)$-category $\mathcal{D}$ has an underlying $\infty$-groupoid $\mathcal{D}^{\sim} \rightarrow \mathcal{D}$, which may be identified with a space. There is a quotient construction as well.

Lemma 7.1. Let $\mathcal{D}$ be an $(\infty, n)$-category. There is an $\infty$-groupoid $|\mathcal{D}|$ and a homomorphism $q: \mathcal{D} \rightarrow|\mathcal{D}|$ so that

(i) every $k$-morphism, $k>0$, in $|\mathcal{D}|$ is invertible, and

(ii) $q: \mathcal{D} \rightarrow|\mathcal{D}|$ is universal with respect to (i).

\footnotetext{
${ }^{10} \mathrm{It}$ is in fact a consequence of the cobordism hypothesis that this space of functions is weakly contractible. This has been proved independently of the cobordism hypothesis in [EM] and also in unpublished work of Galatius.
} 
These constructions are relevant to invertible topological field theories. We say an object $x$ in a symmetric monoidal $(\infty, n)$-category is invertible if it has an inverse $y$ for the monoidal structure: $x \otimes y$ is isomorphic to the unit object.

Definition 7.2. A topological field theory $\alpha: \operatorname{Bord}_{n} \rightarrow \mathcal{C}$ is invertible if $\alpha(W)$ is invertible for all objects and morphisms $W$.

It follows from the cobordism hypothesis that $\alpha$ is invertible if and only if $\alpha\left(\mathrm{pt}_{+}\right)$is invertible. By the universal properties an invertible field theory $\alpha: \operatorname{Bord}_{n} \rightarrow \mathcal{C}$ factors through $\left|\operatorname{Bord}_{n}\right|$ and $\left(\mathcal{C}^{\mathrm{fd}}\right)^{\sim}$ :

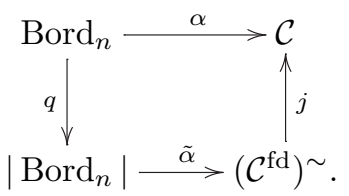

Since $\operatorname{Bord}_{n}$ and $\mathcal{C}$ are symmetric monoidal, so too are $\left|\operatorname{Bord}_{n}\right|$ and $\left(\mathcal{C}^{\mathrm{fd}}\right) \sim$. An $\infty$-groupoid is equivalent to a space (Remark 5.4), and a symmetric monoidal $\infty$ groupoid is equivalent to an infinite loop space, i.e., the 0 -space of a spectrum. Furthermore, $\tilde{\alpha}$ is an infinite loop space map. This reduces the study of invertible topological field theories to a problem in stable homotopy theory.

Remark 7.4. Invertible field theories play a role in ordinary quantum field theory, for example as anomalies.

A corollary of the cobordism hypothesis [L1, §2.5] determines the homotopy type of the spectrum $\left|\operatorname{Bord}_{n}\right|$. Consider first the bordism $(\infty, n)$-category $\operatorname{Bord}_{n}^{\mathrm{fr}}$ of $n$ framed manifolds. The cobordism hypothesis, in the heuristic form Theorem 1.1. asserts that $\operatorname{Bord}_{n}^{\mathrm{fr}}$ is free on one generator. It follows that so too is $\left|\operatorname{Bord}_{n}^{\mathrm{fr}}\right|$. The latter is a spectrum, and the free spectrum on one generator is the sphere spectrum. For the bordism $(\infty, n)$-category of $G$-manifolds $\operatorname{Bord}_{n}^{G}$ the cobordism hypothesis in the form Theorem 6.11 implies that $\left|\operatorname{Bord}_{n}^{G}\right|$ is the $n$th suspension of a MadsenTillmann spectrum. (These spectra are mentioned in 92 before Definition 2.5.)

An $\infty$-groupoid - or $(\infty, 0)$-category - is a model for a space. We may view an $(\infty, n)$-category as a generalization of a space which allows noninvertibility. From that perspective the cobordism hypothesis is a generalization of the Madsen-Weiss theorem.

Variations on the cobordism hypothesis. Morrison and Walker MoW take a somewhat different, but closely related, approach to extended topological field theories which incorporates dualizability from the beginning.

In [L1, §4] Lurie describes several applications and extensions of the cobordism hypothesis. One important extension is to manifolds with singularities, though there are many special cases which do not in fact involve singularities. To illustrate, in Example 5.7 we described a 2-dimensional oriented field theory $F$ associated to a Frobenius algebra $A$. Now suppose that $M$ is a left $A$-module. Recall that $M$ determines a 1-morphism $M: 1 \rightarrow A$ in the Morita 2-category of algebras, where the tensor unit 1 is the trivial algebra $\mathbb{C}$. We might ask what sort of field theory we can associate to the pair $(A, M)$, assuming sufficient finiteness.. A physicist might describe $M$ as giving a boundary condition for $F$, and so extend $F$ to a field theory $\widetilde{F}$ in which some boundaries are colored with the boundary condition $M$. 
For example, a closed interval with one endpoint colored is associated to $M$ as a left $A$-module; the closed interval with both endpoints colored is associated to $M$ as a vector space. The coloring represents a coning off of a point, which is viewed as a manifold with singularities. This is just the tip of the iceberg of possibilities opened up by the cobordism hypothesis with singularities.

From the point of view of algebra, given that $\operatorname{Bord}_{n}^{\mathrm{fr}}$ is the free symmetric monoidal $(\infty, n)$-category with duals on one generator, we might ask how to describe more general symmetric monoidal $(\infty, n)$-categories specified by generators and relations. Roughly speaking, the cobordism hypothesis with singularities identifies these as bordism categories of manifolds with singularities.

Applications to topology. We indicated briefly in Example 5.10 the important role that Chern-Simons theory played in the development of extended topological quantum field theories. That theory encodes invariants of 3-manifolds and links. Newer invariants of links and low-dimensional manifolds were in part inspired by notions in extended field theory. Crane and Frenkel $[\mathrm{CF}$ suggested that categorification of the 3-dimensional invariants would lead to new invariants, potentially related to Donaldson invariants. Later Khovanov $[\mathrm{Kh}$ introduced such a categorification of the Jones polynomial. This now has a proposed derivation from quantum field theory GSV, W4.

There is current research in many directions which will potentially take advantage of more powerful aspects of extended field theories and the cobordism hypothesis in contexts which are not discrete and semisimple. For example, the cobordism hypothesis illuminates string topology invariants and topological versions of Hochschild homology and its cousins [BCT]. It also appears in several discussions of the 2-dimensional extended topological field theories relevant for mirror symmetry: the A-model and the B-model. There is an enormous literature on this subject; see Te2] for one recent example which uses ideas around the cobordism hypothesis.

Applications to algebra. Now we shift focus from topology and bordism categories to the codomain $\mathcal{C}$. Quite generally a homomorphism in algebra organizes the codomain according to the structure of the domain. This principle is often applied in the context of group actions on sets, for example: the structure of orbits and stabilizers illuminates the situation at hand. Here if $F: \operatorname{Bord}_{n} \rightarrow \mathcal{C}$ is a homomorphism, and $F\left(\mathrm{pt}_{+}\right)=x$, then we can study $x$ using smooth manifolds and their gluings.

One application is to $E_{k}$-algebras, which are objects in a symmetric monoidal category which have $k$ associative composition laws. We met $E_{1}$-algebras (ordinary associative algebras) in the category Vect $_{\mathbb{C}}$ of complex vector spaces in Example 5.7. An $E_{2}$-algebra in Vect $_{\mathbb{C}}$ is a commutative algebra and there is nothing higher up: an $E_{k}$-algebra for $k>2$ is also a commutative algebra. More interesting examples are obtained if we look in other symmetric monoidal categories, for example the $\infty$-category of chain complexes. In [L1, §4.1] Lurie describes some relationships between the cobordism hypothesis and $E_{k}$-algebras in $(\infty, n)$-categories. In particular, an $E_{k}$-algebra $A$ in an $(\infty, n)$-category $\mathcal{C}$ is automatically $k$-dualizable, so determines a homomorphism $F: \operatorname{Bord}_{k}^{\mathrm{fr}} \rightarrow E_{k}(\mathcal{C})$, where $E_{k}(\mathcal{C})$ is the $(\infty, n+k)$ category whose objects are $E_{k}$-algebras in $\mathcal{C}$. Thus $E_{k}$-algebras may be studied with smooth manifolds. For example, if $A$ is an ordinary algebra $\left(E_{1}\right.$-algebra), then in the associated field theory $F\left(S^{1}\right)$ is the Hochschild homology of $A$ (see (5.9) for a 
simple example). Since the circle is an $E_{2}$-algebra in the bordism category, so too is the Hochschild homology $F\left(S^{1}\right)$. This assertion is the Deligne conjecture, which together with generalizations is proved in many works, for example [Co, $\overline{\mathrm{KS}}, \mathrm{L2}, \mathrm{BFN}]$. (We remark that there are several other proofs of the Deligne conjecture.)

As another application of the cobordism hypothesis to algebra, we mention ongoing work DHS] which proves that a fusion category [ENO is 3-dualizable. A fusion category is a special type of tensor category, and a tensor category is an $E_{1}$-algebra in the 2-category of linear categories. So tensor categories form a 3category, and it is in that 3-category that fusion categories are fully dualizable. The associated 3-dimensional framed field theory can be brought to bear on the study of fusion categories. We remark that simple topological diagrams involving 0- and 1-dimensional manifolds are usually used to study fusion categories and their cousins. The cobordism hypothesis opens up the possibility of using the more powerful topology of 3-dimensional manifolds. In related ongoing work of the author and Teleman, we consider $E_{2}$-algebras in the 2-category of linear categories; they comprise the 4-category of braided tensor categories. We prove that modular tensor categories are invertible, which now gives a 4-dimensional perspective on quantum groups.

Applications to representation theory. In $\$$ and in Example 5.7 we illustrated a very simple, discrete 2 -dimensional field theory associated to a finite group $G$. There is also a 3-dimensional field theory with values in the 3-category of tensor categories; it attaches the tensor category of vector bundles over $G$ under convolution to pt. (The theory is unoriented - as is the 2-dimensional theory-so we have an unframed unoriented unadorned point.) That theory may be viewed as the simplest case of 3-dimensional Chern-Simons theory (Example 5.10). Ben-Zvi and Nadler [BN] study the analogous theory for a reductive complex group $G$. Discrete categories are futile here; the full force of $\infty$-categories comes into play. One would like a 3-dimensional theory which generalizes that of a finite group, and now attaches the symmetric monoidal $\infty$-category of $\mathcal{D}$-modules on $G$ to a point. However, the necessary finiteness conditions are not satisfied. Instead, they construct a related 2-dimensional field theory, the character theory, which assigns to a point the Hecke category associated to $G$. Then one computes that the category of Lusztig's character sheaves is attached to $S^{1}$. The character theory may be viewed as a dimensional reduction of a 4-dimensional field theory [KW] related to the geometric Langlands program. It seems likely that the topological field theory perspective, and the cobordism hypothesis, will shed light on old questions in the representation theory of semisimple Lie groups.

Echos in quantum field theory. As mentioned earlier, quantum field theorists traditionally only studied 2-tier theories: correlation functions on $n$-manifolds and Hilbert spaces attached to $(n-1)$-manifolds. In recent years the ideas mathematicians have developed around extended field theories, including the cobordism hypothesis, have seeped into physics. In 2-dimensional conformal field theory there is a category of boundary conditions, called $D$-branes, and in topological versions this is understood to be part of an extended field theory. Higher-dimensional analogs are now common; see Kap for a recent review. For example, Kapustin and Witten $\mathrm{KW}$ study a topological twist of the 4-dimensional $N=4$ supersymmetric Yang-Mills theory. Going beyond the traditional two tiers, this theory attaches a 
category to every closed 2-manifold. Kapustin and Witten relate that to a category which appears in the geometric Langlands program. The story is richer: there is a family of theories parametrized by $\mathbb{C P}^{1}$ and $S$-duality acts as an involution on the theories. This suggests an equivalence between two different categories attached to a 2-manifold, which is a topological version of the basic conjecture in the geometric Langlands program.

The maximally supersymmetric $N=4$ Yang-Mills theory is the dimensional reduction of a 6-dimensional supersymmetric field theory which has superconformal invariance. Its name, the $(2,0)$ superconformal field theory in six dimensions, reflects its symmetry group; a simpler name is Theory $\mathscr{X}$. This theory has no classical description. It is predicted to exist from limiting arguments in string theory. Its mysterious nature justifies the appellation Theory $\mathscr{X}$, as does its dimension: si $\mathscr{X}$. A few properties can be predicted from string theory, and these can be used to study compactifications to lower dimensions. Among the many protagonists here we mention Gaiotto $\mathrm{Ga}$ and Gaiotto, Moore and Neitzke GMN. One important ideawhich is clearly inspired by extended field theory and the activity surrounding the cobordism hypothesis - is to study compactifications of the 6-dimensional theory as a function of the compactifying manifold. This is formalized as follows. Suppose $F:$ Bord $_{6} \rightarrow \mathcal{C}$ is a 6 -dimensional extended topological theory. Then for any closed 2-manifold $N$ we obtain a 4-dimensional theory $F_{N}: \operatorname{Bord}_{4} \rightarrow \operatorname{Hom}_{\mathcal{C}}(F(N), F(N))$ defined using Cartesian product $11 F_{N}(M)=F(N \times M)$. Now view $F_{N}$ as a function of $N$. Then we obtain a 2-dimensional extended field theory with values in the $(\infty, 4)$-category of 4 -dimensional field theories! The flexibility in Definition 5.6 which allows arbitrary codomains is heavily used here. One can get other field theories by composing with homomorphisms out of 4-dimensional theories. A recent paper [MoT] implements this idea in a physics context, and it predicts the existence of certain holomorphic symplectic manifolds.

Finally, the renewed interest in $E_{n}$-algebras and their role in extended topological field theories may bring some fresh perspectives to quantum field theories which are not topological. One axiomatic approach to quantum field theory $[\mathrm{H}]$ assigns operator algebras to open sets and describes how they fit together. This idea was imported in an algebro-geometric framework in certain mathematical approaches to 2-dimensional conformal field theory, in vertex operator algebras [Bo] and chiral algebras $\mathrm{BeDr}$. These ideas are circling back to general quantum field theories [CG] with potential to shed new light on their structure.

These are only a few examples of the potential that extended topological field theories and the cobordism hypothesis hold in both mathematics and physics.

\section{About the AUthor}

Dan Freed is professor of mathematics at the University of Texas in Austin. His work in geometry and topology has close connections with quantum field theory and string theory.

\section{ADDED AFTER POSTING}

In the reference DHS of this article Andre Henriques was mistakenly identified as an author. In fact, Noah Snyder is the third author on that forthcoming work.

\footnotetext{
${ }^{11}$ The bordism groups of Pontrjagin and Thom are rings with multiplication given by the Cartesian product. Our discussion of topological field theory has not used this ring structure until now.
} 


\section{REFERENCES}

[A1] M. F. Atiyah, Bordism and cobordism, Proc. Cambridge Philos. Soc. 57 (1961), 200208. MR0126856 (23:A4150)

[A2] _ Topological quantum field theories, Inst. Hautes Études Sci. Publ. Math. (1988), no. 68, 175-186 (1989). MR1001453 (90e:57059)

[Ab] Lowell Abrams, Two-dimensional topological quantum field theories and Frobenius algebras, J. Knot Theory Ramifications 5 (1996) no. 5, 569-587. MR1414088 $(97 \mathrm{j}: 81292)$

[B-N] Dror Bar-Natan, On Khovanov's categorification of the Jones polynomial, Algebr. Geom. Topol. 2 (2002), 337-370 (electronic). MR.1917056 (2003h:57014)

[Ba] Clark Barwick, $(\infty, n)$-Cat as a closed model category, ProQuest LLC, Ann Arbor, MI, 2005. Thesis (Ph.D.)-University of Pennsylvania. MR.2706984

[BC] Martin Betz and Ralph L. Cohen, Graph moduli spaces and cohomology operations, Turkish J. Math. 18 (1994), no. 1, 23-41. MR1270436 (95i:58037)

[BCT] Andrew J. Blumberg, Ralph L. Cohen, and Constantin Teleman, Open-closed field theories, string topology, and Hochschild homology, 0906.5198 MR2581905 (2011f:55020)

[BD] John C. Baez and James Dolan, Higher-dimensional algebra and topological quantum field theory, J. Math. Phys. 36 (1995), no. 11, 6073-6105. MR.1355899 (97f:18003)

[BDH] Arthur Bartels, Christopher L. Douglas, and André G. Henriques, Conformal nets and local field theory, 0912.5307

[Be] Julia E. Bergner, A survey of $(\infty, 1)$-categories Towards higher categories, IMA Vol. Math. Appl., vol. 152, Springer, New York, 2010, pp. 69-83. MR2664620 (2011e:18001)

[BeDr] Alexander Beilinson and Vladimir Drinfeld, Chiral algebras, American Mathematical Society Colloquium Publications, vol. 51, American Mathematical Society, Providence, RI, 2004. MR2058353 (2005d:17007)

[BFN] David Ben-Zvi, John Francis, and David Nadler, Integral transforms and Drinfeld centers in derived algebraic geometry, J. Amer. Math. Soc. 23 (2010), no. 4, 909966. MR2669705 (2011j:14023)

[BN] David Ben-Zvi and David Nadler, The Character Theory of a Complex Group, 0904.1247, preprint.

[Bo] Richard E. Borcherds, Vertex algebras, Kac-Moody algebras, and the Monster, Proc. Nat. Acad. Sci. U.S.A. 83 (1986), no. 10, 3068-3071. MR843307 (87m:17033)

[BS] Clark Barwick and Christopher Schommer-Pries, On the Unicity of the Homotopy Theory of Higher Categories, 1112.0040

[C] Jean Cerf, La stratification naturelle des espaces de fonctions différentiables réelles et le théorème de la pseudo-isotopie, Inst. Hautes Études Sci. Publ. Math. (1970), no. 39, 5-173. MR0292089 (45:1176)

[CF] Louis Crane and Igor B. Frenkel, Four-dimensional topological quantum field theory, Hopf categories, and the canonical bases, J. Math. Phys. 35 (1994) no. 10, 51365154. Topology and physics. MR.1295461 (96d:57019)

[CG] K. Costello and O. Gwilliam, Factorization algebras in perturbative quantum field theory. http://math.northwestern.edu/ costello/factorization_public.html in preparation.

[Co] Kevin Costello, Topological conformal field theories and Calabi-Yau categories, Adv. Math. 210 (2007) no. 1, 165-214. MR2298823 (2008f:14071)

[CS] Moira Chas and Dennis Sullivan, String Topology, math/9911159.

[D] S. K. Donaldson, Polynomial invariants for smooth four-manifolds, Topology 29 (1990), no. 3, 257-315. MR1066174 (92a:57035)

[Da] O. Davidovich, State sums in 2-dimensional fully extended topological field theories, Ph.D. thesis, University of Texas at Austin, 2011. http://repositories. lib.utexas.edu/handle/2152/ETD-UT-2011-05-3139.

[DHS] C. Douglas, A. Henriques, and C. Schommer-Pries, The 3-category of tensor categories. in preparation. 
[Di] R. Dijkgraaf, A geometrical approach to two-dimensional conformal field theory, Ph.D. thesis, University of Utrecht, 1989. http://igitur-archive. library.uu.nl/dissertations/2011-0929-200347/UUindex.html.

[DW] Robbert Dijkgraaf and Edward Witten, Topological gauge theories and group cohomology, Comm. Math. Phys. 129 (1990), no. 2, 393-429. MR1048699 (91g:81133)

[EM] Yakov M. Eliashberg and Nikolai M. Mishachev, The space of framed functions is contractible, 1108.1000

[ENO] Pavel Etingof, Dmitri Nikshych, and Viktor Ostrik, On fusion categories, Ann. of Math. (2) 162 (2005), no. 2, 581-642. MR2183279 (2006m:16051)

[F] Daniel S. Freed, Higher algebraic structures and quantization, Comm. Math. Phys. 159 (1994), no. 2, 343-398, arXiv:hep-th/9212115 MR.1256993 (95c:58034)

[Fa] L. Faddeev, Elementary introduction to quantum field theory, Quantum fields and strings: a course for mathematicians. Vol. 1 (P. Deligne et. al., ed.), American Mathematical Society, 1999, pp. 513-550. Notes by L. Jeffrey. MR.1701606 (2002a:81154)

[FHLT] Daniel S. Freed, Michael J. Hopkins, Jacob Lurie, and Constantin Teleman, Topological quantum field theories from compact Lie groups, A celebration of the mathematical legacy of Raoul Bott, CRM Proc. Lecture Notes, vol. 50, Amer. Math. Soc., Providence, RI, 2010, pp. 367-403. arXiv:0905.0731 MR 2648901 (2011i:57040)

[FHT] Daniel S. Freed, Michael J. Hopkins, and Constantin Teleman, Consistent orientation of moduli spaces, The many facets of geometry, Oxford Univ. Press, Oxford, 2010, pp. 395-419. arXiv:0711.1909. MR2681705(2011h:57043)

[FQ] Daniel S. Freed and Frank Quinn, Chern-Simons theory with finite gauge group, Comm. Math. Phys. 156 (1993), no. 3, 435-472, arXiv:hep-th/911100 MR.1240583 (94k:58023)

[G] E. Getzler, Batalin-Vilkovisky algebras and two-dimensional topological field theories, Comm. Math. Phys. 159 (1994), no. 2, 265-285. MR 1256989 (95h:81099)

[Ga] Davide Gaiotto, $N=2$ dualities, JHEP 1208 (2011), 034, arXiv:0904.2715

[GJ] James Glimm and Arthur Jaffe, Quantum physics, second ed., Springer-Verlag, New York, 1987. A functional integral point of view. MR887102 (89k:81001)

[GMN] Davide Gaiotto, Gregory W. Moore, and Andrew Neitzke, Wall-crossing, Hitchin Systems, and the WKB Approximation, 0907.3987

[GMTW] Søren Galatius, Ulrike Tillmann, Ib Madsen, and Michael Weiss, The homotopy type of the cobordism category, Acta Math. 202 (2009) no. 2, 195-239, arXiv:math/0605249 MR2506750(2011c:55022)

[GSV] Sergei Gukov, Albert Schwarz, and Cumrun Vafa, Khovanov-Rozansky homology and topological strings, Lett. Math. Phys. 74 (2005), no. 1, 53-74. MR2193547 (2007a:57014)

[H] Rudolf Haag, Local quantum physics, second ed., Texts and Monographs in Physics, Springer-Verlag, Berlin, 1996. Fields, particles, algebras. MR1405610 (98b:81001)

[I] Kiyoshi Igusa, The space of framed functions, Trans. Amer. Math. Soc. 301 (1987), no. 2, 431-477. MR882699 (88g:57034)

[K] Robion Kirby, A calculus for framed links in $S^{3}$, Invent. Math. 45 (1978), no. 1, 35-56. MR0467753 (57:7605)

[Ka] Daniel M. Kan, Adjoint functors, Trans. Amer. Math. Soc. 87 (1958), 294-329. MR.0131451(24:A1301)

[Kap] Anton Kapustin, Topological field theory, higher categories, and their applications, Proceedings of the International Congress of Mathematicians. Volume III (New Delhi), Hindustan Book Agency, 2010, pp. 2021-2043.arXiv:1004:2307 MR2827874

[Kh] Mikhail Khovanov, A categorification of the Jones polynomial, Duke Math. J. 101 (2000), no. 3, 359-426. MR1740682(2002j:57025)

[KM] M. Kontsevich and Yu. Manin, Gromov-Witten classes, quantum cohomology, and enumerative geometry, Comm. Math. Phys. 164 (1994), no. 3, 525-562, arXiv:hep-th/9402147 MR1291244 (95i:14049)

[Ko] Joachim Kock, Frobenius algebras and $2 D$ topological quantum field theories, London Mathematical Society Student Texts, vol. 59, Cambridge University Press, Cambridge, 2004. MR2037238(2005a:57028)

[KS] M. Kontsevich and Y. Soibelman, Notes on $A_{\infty}$-algebras, $A_{\infty}$-categories and noncommutative geometry, Homological mirror symmetry, Lecture Notes in Phys., 
vol. 757, Springer, Berlin, 2009, pp. 153-219. arXiv:math/0606241 MR2596638 (2011f:53183)

[KW] Anton Kapustin and Edward Witten, Electric-magnetic duality and the geometric Langlands program, Commun. Number Theory Phys. 1 (2007), no. 1, 1-236, arXiv:hep-th/0604151 MR2306566 (2008g:14018)

[L1] Jacob Lurie, On the classification of topological field theories, Current developments in mathematics, 2008, Int. Press, Somerville, MA, 2009, pp. 129-280. arXiv:0905.0465 MR2555928(2010k:57064)

[L2] _ Higher Algebra. http://math.harvard.edu/ lurie/papers/higheralgebra. pdf. draft version.

[L3] Lectures on the cobordism hypothesis. http://www.ma.utexas.edu/video/ perspectives.html. Perspectives in Geometry lectures at University of Texas, January, 2009.

[LZ] Bong H. Lian and Gregg J. Zuckerman, New perspectives on the BRST-algebraic structure of string theory, Comm. Math. Phys. 154 (1993), no. 3, 613-646. MR:1224094 (94e:81333)

[Ma] George W. Mackey, The mathematical foundations of quantum mechanics: A lecturenote volume, W., A. Benjamin, Inc., New York-Amsterdam, 1963. MR676642 (84g:81003)

[May] J. P. May, Stable algebraic topology, 1945-1966, History of topology, North-Holland, Amsterdam, 1999, pp. 665-723.

[Mc] Saunders Mac Lane, Categories for the working mathematician, second ed., Graduate Texts in Mathematics, vol. 5, Springer-Verlag, New York, 1998. MR 0354798 (50:7275)

[Mi1] John W. Milnor, Topology from the differentiable viewpoint, Based on notes by David W. Weaver, The University Press of Virginia, Charlottesville, Va., 1965. MR 0226651 $(37: 2239)$

[Mi2] Morse theory, Based on lecture notes by M. Spivak and R. Wells. Annals of Mathematics Studies, No. 51, Princeton University Press, Princeton, N.J., 1963. MR.0163331(29:634)

[Mi3] Lectures on the h-cobordism theorem, Notes by L. Siebenmann and J. Sondow, Princeton University Press, Princeton, N.J., 1965. MR0190942 (32:8352)

[MoT] Gregory W. Moore and Yuji Tachikawa, On $2 d$ TQFTs whose values are holomorphic symplectic varieties, 1106.5698

[MoW] Scott Morrison and Kevin Walker, Higher categories, colimits, and the blob complex, Proc. Natl. Acad. Sci. USA 108 (2011), no. 20, 8139-8145, arXiv:1108.5386. MR.2806651 (2012f:18019)

[MS] Gregory W. Moore and Graeme B. Segal, Dirichlet branes and mirror symmetry, Clay Mathematics Monographs, vol. 4, ch. D-branes and $K$-theory in $2 D$ conformal field theory, pp. $\mathrm{x}+681$, American Mathematical Society, Providence, RI, 2009. arXiv:hep-th/0609042 MR2567952 (2011e:53148)

[MSei] Gregory W. Moore and Nathan Seiberg, Classical and quantum conformal field theory, Comm. Math. Phys. 123 (1989), no. 2, 177-254. MR.1002038 (90e:81216)

[MT] Ib Madsen and Ulrike Tillmann, The stable mapping class group and $Q\left(\mathbb{C P}_{+}^{\infty}\right)$, Invent. Math. 145 (2001) no. 3, 509-544. MR1856399 (2002h:55011)

[MW] Ib Madsen and Michael Weiss, The stable moduli space of Riemann surfaces: Mumford's conjecture, Ann. of Math. (2) 165 (2007) no. 3, 843-941, arXiv:math/0212321 MR2335797 (2009b:14051)

[Mu] David Mumford, Towards an enumerative geometry of the moduli space of curves, Arithmetic and geometry, Vol. II, Progr. Math., vol. 36, Birkhäuser Boston, Boston, MA, 1983, pp. 271-328. MR717614 (85j:14046)

[P] L. S. Pontryagin, Smooth manifolds and their applications in homotopy theory, American Mathematical Society Translations, Ser. 2, Vol. 11, American Mathematical Society, Providence, R.I., 1959, pp. 1-114. MR0115178(22:5980)

[Pa] Richard S. Palais, Seminar on the Atiyah-Singer index theorem, With contributions by M. F. Atiyah, A. Borel, E. E. Floyd, R. T. Seeley, W. Shih and R. Solovay. Annals of Mathematics Studies, No. 57, Princeton University Press, Princeton, N.J., 1965. MR0198494(33:6649) 
[Q] Frank Quinn, Lectures on axiomatic topological quantum field theory, Geometry and quantum field theory (Park City, UT, 1991), IAS/Park City Math. Ser., vol. 1, Amer. Math. Soc., Providence, RI, 1995, pp. 323-453. MR1338394 (96e:57021)

[R] Charles Rezk, A Cartesian presentation of weak n-categories, Geom. Topol. 14 (2010), no. 1, 521-571, arXiv:0901.3602. MR2578310 (2010m:18005)

[RT1] N. Yu. Reshetikhin and V. G. Turaev, Ribbon graphs and their invariants derived from quantum groups, Comm. Math. Phys. 127 (1990), no. 1, 1-26. MR1036112 (91c:57016)

[RT2] N. Reshetikhin and V. G. Turaev, Invariants of 3-manifolds via link polynomials and quantum groups, Invent. Math. 103 (1991) no. 3, 547-597. MR1091619(92b:57024)

[Se1] G. B. Segal, Felix Klein Lectures 2011. http://www.mpim-bonn.mpg.de/node/3372/ abstracts.

[Se2] The definition of conformal field theory, Topology, geometry and quantum field theory, London Math. Soc. Lecture Note Ser., vol. 308, Cambridge Univ. Press, Cambridge, 2004, pp. 421-577. MR2079383(2005h:81334)

[Se3] _ String algebras. http://www.cgtp.duke.edu/ITP99/segal/stanford/ lect5.pdf.

[Sm] S. Smale, On the structure of manifolds, Amer. J. Math. 84 (1962), 387-399. MR0153022 (27:2991)

[Sp] Edwin H. Spanier, Algebraic topology, Springer-Verlag, New York, 1981. Corrected reprint. MR666554 (83i:55001)

[SW] R. F. Streater and A. S. Wightman, PCT, spin and statistics, and all that, Princeton Landmarks in Physics, Princeton University Press, Princeton, NJ, 2000. Corrected third printing of the 1978 edition. MR,1884336 (2003f:81154)

[T] René Thom, Quelques propriétés globales des variétés différentiables, Comment. Math. Helv. 28 (1954), 17-86. MR0061823 (15:890a)

[Te1] C. Teleman, Five lectures on topological field theory. http://math.berkeley. edu/ teleman/math/barclect.pdf. MR2648326(2011d:57078)

[Te2] Lie group actions in categories and Langlands duality. http://www.ma. utexas.edu/video/perspectives.html. Perspectives in Geometry lectures at University of Texas, October, 2011.

[Til1] Ulrike Tillmann, The classifying space of the $(1+1)$-dimensional cobordism category, J. Reine Angew. Math. 479 (1996), 67-75. MR.1414388 (97i:55035)

[Til2] On the homotopy of the stable mapping class group, Invent. Math. 130 (1997) no. 2, 257-275. MR1474157 (99k:57036)

[Tu] V. G. Turaev, Quantum invariants of knots and 3-manifolds, de Gruyter Studies in Mathematics, vol. 18, Walter de Gruyter \& Co., Berlin, 1994 . MR1292673 (95k:57014)

[W1] Edward Witten, Topological quantum field theory, Comm. Math. Phys. 117 (1988), no. 3, 353-386. MR953828 (89m:57037)

[W2] Topological sigma models, Comm. Math. Phys. 118 (1988), no. 3, 411-449. MR.958805 (90b:81080)

[W3] Quantum field theory and the Jones polynomial, Comm. Math. Phys. 121 (1989), no. 3, 351-399. MR990772 (90h:57009)

[W4] Fivebranes and knots, Quantum Topol. 3 (2012), no. 1, 1-137, arXiv:1101. 3216. MR2852941

[Wa] K. Walker, On Witten's 3-manifold invariants. http://canyon23.net/math/ 1991TQFTNotes.pdf.

The University of Texas at Austin, Mathematics Department RLM 8.100, 2515 SpeedWay Stop C1200, Austin, Texas 78712-1202

E-mail address: dafr@math.utexas.edu 